\title{
Surface tensions of multi-component mixed inorganic/organic aqueous systems of atmospheric significance: measurements, model predictions and importance for cloud activation predictions
}

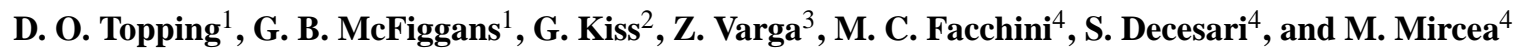 \\ ${ }^{1}$ School of Earth, Atmospheric and Environmental Sciences, The University of Manchester, The Sackville street building, \\ Sackville street, Manchester, M60 1QD, UK \\ ${ }^{2}$ Air Chemistry Group of the Hungarian Academy of Sciences, Univ. of Pannonia, 8201Veszprém, P.O. Box 158, Hungary \\ ${ }^{3}$ Department of Earth and Environmental Sciences, University of Pannonia, 8201 Veszprém, P.O. Box 158, Hungary \\ ${ }^{4}$ Instituto di Scienze dell Atmosfera e del Clima, Consiglio Nazionale delle Ricerche, Via Gobetti, Bologna, Italy
}

Received: 6 November 2006 - Published in Atmos. Chem. Phys. Discuss.: 27 November 2006

Revised: 8 March 2007 - Accepted: 24 April 2007 - Published: 10 May 2007

\begin{abstract}
In order to predict the physical properties of aerosol particles, it is necessary to adequately capture the behaviour of the ubiquitous complex organic components. One of the key properties which may affect this behaviour is the contribution of the organic components to the surface tension of aqueous particles in the moist atmosphere. Whilst the qualitative effect of organic compounds on solution surface tensions has been widely reported, our quantitative understanding on mixed organic and mixed inorganic/organic systems is limited. Furthermore, it is unclear whether models that exist in the literature can reproduce the surface tension variability for binary and higher order multi-component organic and mixed inorganic/organic systems of atmospheric significance. The current study aims to resolve both issues to some extent. Surface tensions of single and multiple solute aqueous solutions were measured and compared with predictions from a number of model treatments. On comparison with binary organic systems, two predictive models found in the literature provided a range of values resulting from sensitivity to calculations of pure component surface tensions. Results indicate that a fitted model can capture the variability of the measured data very well, producing the lowest average percentage deviation for all compounds studied. The performance of the other models varies with compound and choice of model parameters. The behaviour of ternary mixed inorganic/organic systems was unreliably captured by using a predictive scheme and this was dependent on the composition of the solutes present. For more atmospherically representative higher order systems, entirely predictive schemes performed poorly. It was found that use of the binary data in
\end{abstract}

Correspondence to: D. O. Topping

(david.topping@manchester.ac.uk) a relatively simple mixing rule, or modification of an existing thermodynamic model with parameters derived from binary data, was able to accurately capture the surface tension variation with concentration. Thus, it would appear that in order to model multi-component surface tensions involving compounds used in this study one requires the use of appropriate binary data. However, results indicate that the use of theoretical frameworks which contain parameters derived from binary data may predict unphysical behaviour when taken beyond the concentration ranges used to fit such parameters. The effect of deviations between predicted and measured surface tensions on predicted critical saturation ratios was quantified, by incorporating the surface tension models into an existing thermodynamic framework whilst firstly neglecting bulk to surface partitioning. Critical saturation ratios as a function of dry size for all of the multi-component systems were computed and it was found that deviations between predictions increased with decreasing particle dry size. As expected, use of the surface tension of pure water, rather than calculate the influence of the solutes explicitly, led to a consistently higher value of the critical saturation ratio indicating that neglect of the compositional effects will lead to significant differences in predicted activation behaviour even at large particle dry sizes. Following this two case studies were used to study the possible effect of bulk to surface partitioning on critical saturation ratios. By employing various assumptions it was possible to perform calculations not only for a binary system but also for a mixed organic system. In both cases this effect lead to a significant increase in the predicted critical supersaturation ratio compared to the above treatment. Further analysis of this effect will form the focus of future work.

Published by Copernicus Publications on behalf of the European Geosciences Union. 


\section{Introduction}

The complex behaviour of multi-component aerosol particles in the moist atmosphere requires predictive frameworks which attempt to capture the complexity of the organic composition and its combination with inorganic compounds. There are however numerous restrictions hindering the construction of such frameworks across the entire model hierarchy. Solely focussing on the bulk hygroscopic behaviour of the atmospheric aerosol, these restrictions include the need for computational efficiency (e.g. Amundson et al., 2006; Metzger et al., 2002), lack of appropriate laboratory data (e.g. Clegg et al., 2001; Marcolli et al., 2004; Peng et al., 2001) and even difficulty in constructing "complete" theoretical frameworks (e.g. Clegg and Seinfeld, 2006; Clegg et al., 2001). The equilibrium behaviour of aqueous systems is treated in the same manner whether making predictions of warm cloud activation or growth in the sub-saturated humid regime. Neglecting the influence of any solid-air interface, the equilibrium relationship for water is given as Eq. (1):

$$
\frac{\mathrm{RH}}{100 \%}=a_{w} \exp \left(\frac{2 v_{w} \sigma_{w s}}{R T r_{\mathrm{drop}}}\right)
$$

where $a_{w}$ is the water activity, $v_{w}$ the partial molar volume of water $\left(\mathrm{m}^{3} \mathrm{~mol}^{-1}\right), \sigma_{w s}$ the solution surface tension $\left(\mathrm{N} \mathrm{m}^{-1}\right)$, $R$ the universal gas constant $\left(8.314 \mathrm{~J} \mathrm{~K}^{-1} \mathrm{~mol}^{-1}\right), T$ the temperature (K) and $r_{\text {drop }}$ the radius of the droplet (m). Whilst the formulation, known as the "primitive" Kohler equation (see McFiggans et al., 2006), appears relatively simple, capturing the composition dependence of the relevant parameters is frequently hindered by the complexity of the organic fraction. Similarly there is difficulty when attempting to couple this with inorganic compounds. The challenges encountered in modelling the solute effect $\left(a_{w}\right)$ have been reviewed in the literature to varying extents (e.g. Clegg et al., 2001; Ming and Russell, 2002; Topping et al., 2005b). However, the complexities involved with calculating the Kelvin effect (exponential term) have received less attention, although the composition dependence and potential importance of this term has been highlighted by both theoretical (e.g. Feingold, 2003; McFiggans et al., 2006; Rissman et al., 2004) and references therein) and analytical studies alike (Shulman et al., 1996; Sorjamaa et al., 2004; Kiss et al., 2005).

The Raoult effect, encompassed in the water activity $a_{w}$ in Eq. (1), describes the influence of the solute on the equilibrium vapour pressure above solution, the solution becoming more ideal as the droplet approaches activation. The Kelvin equation, given by the exponential term, tells us that the vapour pressure over a curved interface always exceeds that of the same substance over a flat surface. Thus the effect of the liquid/gas interface is captured in the surface tension and curvature term in Eq. (1). Whilst other composition dependent parameters exist in the exponential term, a primary requirement when incorporating the Kelvin effect into an aerosol model is a method for calculating the surface ten- sion. This must be related to the aerosol composition in a predictive framework. For single component aerosols one can simply use empirical data; yet in mixed solutions, a problem analogous to that encountered in dealing with activities arises. Since the atmospheric aerosol is a complex mixture of inorganic and organic compounds, an appropriate formalism for calculating multi-component surface tension must be used. Similarly, composition dependent variability in ambient samples is likely to depend on sampling location and the history of the aerosol.

Kiss et al. (2005) discuss the relative merits of the two main approaches that have been used to represent the organic fraction of the atmospheric aerosol. The first involves calculations with input data obtained from experiments using model compounds, the second relating to the use of calculations deduced from real ambient samples without an "exact" knowledge of the organic composition. In either case, validation of the use of 'model compounds' or analysing the breadth of surface tension variability not captured in selected ambient measurements requires the use of robust surface tension models. Previously reported models rely on parameters estimated from laboratory measurements. It is necessary to investigate whether it is possible to make use of such approaches to build a predictive framework for atmospheric applications.

There have been previous attempts to model the surface tension of mixed systems using representative species. Tuckermann and Cammenga (2004) measured the surface tensions of aqueous solutions of levoglucosan, 3hydroxybutanoic acid, 3-hydroxybenzoic acid, azelaic acid, pinonic acid and humic acid. Using different relative contributions from the above components, one for a mixture composition suggested to be representative of atmospheric water soluble organic carbon (WSOC) as given by Fuzzi et al. (2001), the authors found that a simple linear combination of the surface tension reduction by the single model compounds could reproduce the mixture behaviour very well. However, comparison of such results with data for real cloud and fog water, as provided by Facchini et al. (2000), showed that the surface tension of the representative mixture did not reproduce the larger surface tension depression from the ambient samples. As pointed out by Tuckermann and Cammenga (2004), it may be that the ambient aerosols contained species not detected in the analysis of the WSOC. Seidl (2000) suggested the influence of insoluble surfactants such as stearic acid which may form a film at the air-solution interface, thus further reducing the surface tension. On the other hand the reductions in surface tension induced by organic compounds strongly depend on the $\mathrm{pH}$-values and on the concentrations of inorganic compounds in the aqueous solutions (Tuckermann and Cammenga, 2004). Tuckermann and Cammenga (2004) used humic acid rather than the suggested proxy for humic like material: Suwannee river fulvic acid. This may introduce further inconsistencies since Brooks et al. (2004) found that the water uptake behaviour of 
Table 1a. Organic aqueous binary systems studied, along with the range of solute mass fractions and derived parameters for use with the binary surface tension model described in Sect. 3.2.1.

\begin{tabular}{|c|c|c|c|c|c|c|c|}
\hline \multirow[t]{3}{*}{ Compound } & \multirow{3}{*}{$\begin{array}{l}\text { Molecular } \\
\text { weight }\end{array}$} & \multicolumn{4}{|c|}{ Binary solute mass fraction experimental range } & \multirow[b]{3}{*}{$\Gamma_{i}^{\psi w O}$} & \multirow[b]{3}{*}{$k_{i}$} \\
\hline & & \multirow{2}{*}{$\begin{array}{l}\text { Source } 1 \\
\text { Max }\end{array}$} & \multirow[b]{2}{*}{ Min } & \multirow{2}{*}{\begin{tabular}{|l|} 
Source 2 \\
Max
\end{tabular}} & \multirow[b]{2}{*}{ Min } & & \\
\hline & & & & & & & \\
\hline Levoglucosan & 162.14 & 0.278707 & 0.008669 & & & 652182717.2 & $7.9761708 \mathrm{~d}-10$ \\
\hline Oxalic acid & 90.03 & 0.095238 & 0.000692 & 0.063692 & 0.001041 & 0.00368646 & 25.51892617 \\
\hline Pinonic acid & 184.23 & 0.005742 & 0.00009 & & & 0.00369131 & 72.65341779 \\
\hline Glutaric acid & 132.11 & 0.52381 & 0.000678 & & & 0.00296865 & 139.4950309 \\
\hline Succinic acid & 118.09 & 0.074074 & 0.000087 & 0.02441 & 0.000875 & 0.00304052 & 69.42952191 \\
\hline Suwannee River fulvic acid & $732.596^{\$}$ & 0.009263 & 0.000019 & & & 0.00259134 & 35942.0372 \\
\hline Malonic acid & 104.06 & & & 0.406191 & 0.000929 & 0.00055578 & 2108.971823 \\
\hline Maleic acid & 116.07 & & & 0.402733 & 0.0009 & 0.00163897 & 293.1181882 \\
\hline Malic acid & 134.09 & & & 0.404468 & 0.000948 & 0.00059786 & 2123.992313 \\
\hline Adipic acid & 146.14 & & & 0.010875 & 0.000789 & 0.00078035 & 2260.690784 \\
\hline Citric acid & 192.12 & & & 0.388823 & 0.000821 & 0.0034211 & 1093655.214 \\
\hline \multicolumn{8}{|c|}{ Unless specified all molecular weights taken from the NIST database } \\
\hline \multicolumn{8}{|c|}{ \$- calculated based on the chemical structure } \\
\hline \multicolumn{8}{|c|}{ For Suwanee River fulvic acid the structure used as discussed by Topping et al 2005b } \\
\hline & & & & & & & \\
\hline measured & Insit & en: & nosfe & $\mathrm{Cl}$ & & & \\
\hline
\end{tabular}

Table 1b. Ternary and higher order systems studied along with the relative percentage contribution from each relevant compound. Also given are the maximum and minimum solute mass fractions for which experimental data was taken.

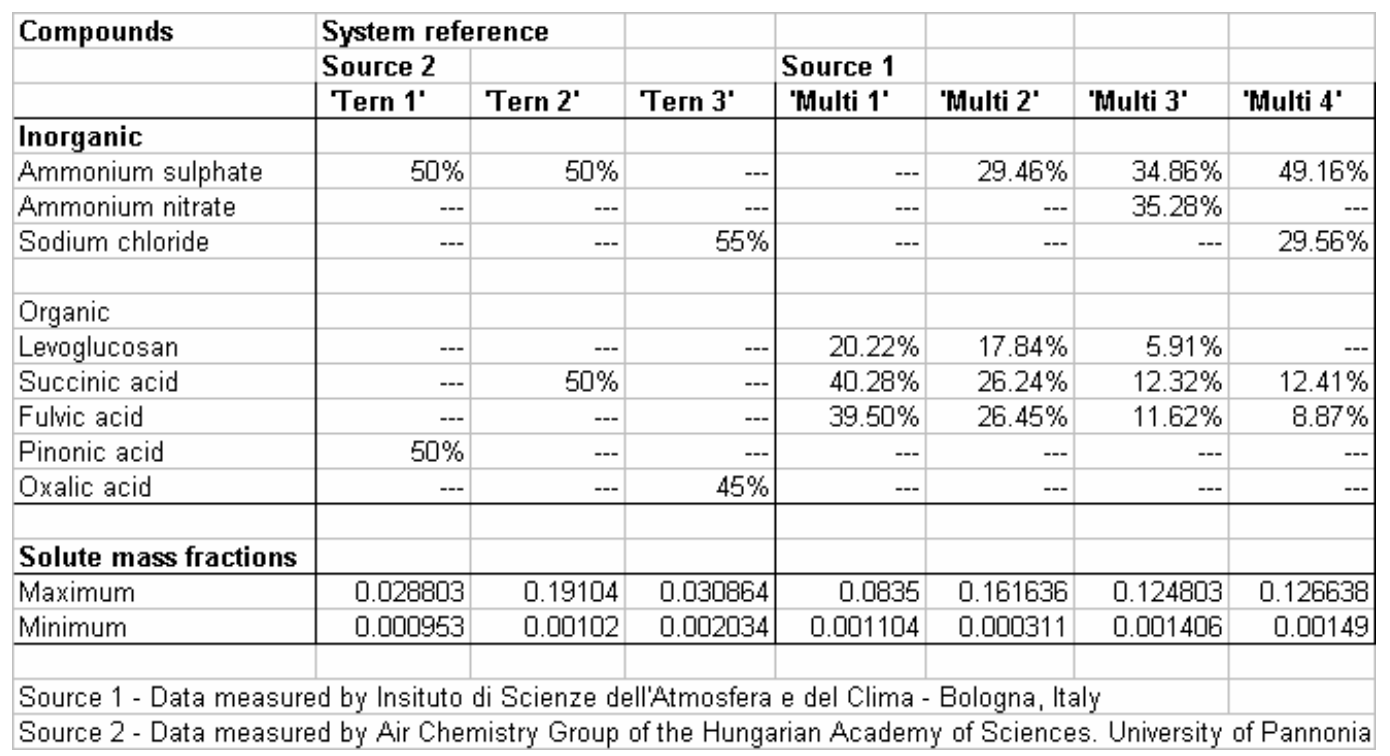

humic like material varies with the type, source, and isolation method. Henning et al. (2005) measured the surface tensions of mixtures including sodium chloride, adipic acid and succinic acid at concentrations encountered around the critical saturation ratio. Here the authors modified the SzyskowskiLangmuir equation presented by Facchini et al. (2000) to make it applicable to mixtures and found good agreement.

In this study an analysis of more complex mixtures is made using individually suggested atmospherically relevant species. By combining a variety of modelling approaches found in the literature and new experimental data the aim is to further improve our understanding on the ability to capture the variation in surface tension with composition. Firstly, binary and ternary organic and mixed inorganic/organic systems are analysed. Following this, analysis of higher order multi-component organic and mixed inorganic/organic systems is made using existing and extended modelling frameworks developed in this body of work. All systems studied and the relative compositions are given in Table $1 \mathrm{a}$ and $1 \mathrm{~b}$, the experimental setup and reasoning behind choice of compounds discussed in the following section. 


\section{Experimental}

\subsection{Preparation of binary and multicomponent systems}

Binary organic systems were prepared by dissolving a quantity of the pure compound in milliQ water providing a maximum concentration which is 1.2 to 2 times lower than that of the saturated solution. In the case of levoglucosan, for which no solubility data are available, a maximum concentration of $28 \% \mathrm{w} / \mathrm{w}$ was studied. Diluted solutions were prepared for each standard in order to measure the surface tension over four orders of magnitude of bulk concentration. The organic binary systems as presented in Table 1a were chosen among the most common ones considered relevant for modelling the atmospheric aerosol hygroscopic properties and CCN ability (e.g. Hori et al., 2003; Chan et al., 2005). Such systems include a polyol (levoglucosan) which is a major product of biomass burning, and pinonic acid which is an aliphatic oxo-carboxylic acid of biogenic origin. The other low-molecular weight carboxylic acids include C2-C6 diacids from those most commonly produced by anthropogenic or biogenic sources, spanning a wide range of water solubilities and with different hydrophobic characters. A very soluble C6 tricarboxylic acid, namely citric acid, and a slightly soluble polycarboxylic acid, the Suwannee River fulvic acid, were also considered. The latter is so far the best surrogate for the complex humic-like substances which are ubiquitous component of the organic aerosols (Fuzzi et al., 2001; Alfarra et al., 2006). Pinonic acid is the wellknown product of terpene oxidation, which is why it was also included in our experiments and other studies (e.g. Shulman et al., 1996). Ammonium sulphate and $\mathrm{NaCl}$ are representative components in continental and marine aerosol, respectively. The ability of models chosen in this study to reproduce the behaviour of such systems is discussed in Sect. 4.1. Moving on from binary systems, ternary systems comprising one inorganic and one organic solute were studied. These solutions comprised $\left(\mathrm{NH}_{4}\right)_{2} \mathrm{SO}_{4}$ :Pinonic acid, $\left(\mathrm{NH}_{4}\right)_{2} \mathrm{SO}_{4}$ :Succinic acid and $\mathrm{NaCl}$ :Oxalic acid at mass ratios of 50:50, 50:50 and 55:45 respectively as presented in Table 1b. Organic compounds account for a significant part (half or even more) of the aerosol mass in various environments but the surface tension effect may also be influenced by inorganic salts when present in relatively high concentration so it is important to analyse the combined effect for ternary and higher order systems. With regards to the multicomponent systems, a mixed system consisting of only organic compounds was studied separately (Table 1b). The mixed system ("Multi 1") includes three organic compounds, namely levoglucosan, succinic acid and fulvic acid, selected to represent chemical classes which are commonly found in the water-soluble fraction of the aerosol, i.e., polyhydroxylated compounds, low-molecular weight aliphatic carboxylic acids, and humic-like substances (Svenningsson et al., 2006). Similarly, three mixed inorganic/organic systems were stud- ied as presented in Table 1b. The mixed systems include the three organic compounds of "Multi 1", plus a variable amount of inorganic ionic material, ranging from 30 to $78 \%$ of the total solute mass. The inorganic salts are selected from the most common and well-known inorganic constituents of atmospheric aerosol particles: ammonium sulfate (for all three mixed systems), ammonium nitrate (in "Multi 3") and sodium chloride (in "Multi 4") (Svenningsson et al., 2006). The ability of models chosen in this study to reproduce the behaviour of such systems is discussed in Sects. 4.3 and 4.4. The maximum solute concentration of the multi-component systems was such that the concentrations of the least soluble compounds (succinic acid and fulvic acid) were just below their solubility limits. Again, diluted solutions were prepared in order to be able to measure the surface tension over three to four orders of magnitude of solute concentrations.

\subsection{Surface tension measurements}

Axisymmetric drop analysis (ADSA) was used to determine surface tension on a drop of sample (Loglio et al., 2001). The method allows surface tension measurement of an aqueous sample on the basis of the drop geometry, and for concentrated solutions the density of the liquid. ADSA was performed at the Institute of Atmospheric Science and Climate (ISAC) by a SINTECH (Berlin, Germany) PAT1 tensiometer. The instruments consists of: 1) a dosing system with a Hamilton syringe connected to the capillary to form a pendent drop, 2) a video camera with a lens, and 3) a frame grabber to transfer the image to a computer. Drops of $11-15 \mathrm{~mm}^{3}$ were formed at the edge of a capillary of $2 \mathrm{~mm}$ external diameter. The volume was held constant for $30 \mathrm{~min}$ after drop formation to allow surface tension to equilibrate. An analogous experimental apparatus was deployed by the Air Chemistry group of the Hungarian Academy of Science, University of Pannonia, providing the surface tension data for additional binary organic systems (Table 1a). In this instance however a FTA 125 (First Ten Angstroms, USA) tensiometer was used.

\section{Modelling}

In the following section a variety of modelling approaches applicable to both inorganic/organic and mixed inorganic/organic systems are discussed.

\subsection{Inorganic systems}

Inorganic systems appear to be relatively well understood and as such are only briefly discussed here. For multicomponent systems, three groups of calculation have been presented in the literature (Hu and Lee, 2004). These include simple additive methods and conventional methods based on the Gibbs adsorption equation and are briefly discussed by Topping et al. (2005a). A common approach consists combining thermodynamic relations together with an adsorption 
model, thus accounting for transfer of material between the bulk and a surface phase, altering molecular forces and the subsequent surface tension. With regards to the latter consideration, in the model presented by Li and Lu (2001), the authors combined the Gibbs dividing surface, the Langmuir adsorption equation and an appropriate model for calculating activity coefficients in mixed solutions to arrive at two different schemes. Briefly, the Gibbs dividing surface is a geometrical surface used to define the volumes of the bulk and surface phases. The Langmuir adsorption isotherm is a relationship which describes the number of adsorbed molecules on a surface to the concentration above that surface. For more information on this model the reader is referred to Seinfeld and Pandis (1998). The basic scheme for binary systems, derived and discussed in more detail in Sect. 3.2.1, is given as Eq. (2):

$\sigma_{w s}=\sigma_{w}+R T \Gamma_{i}^{w o} \ln \left(\frac{1}{1+\mathrm{K}_{i} a_{i}}\right)$

Where $\sigma_{w s}$ is the surface tension of the mixture, $\sigma_{w}$ is the surface tension of pure water, $\Gamma_{i}^{w o}$ is the saturated surface excess of solute " $i$ " ( $\mathrm{mol} \mathrm{m}{ }^{-2}$ tabulated), $k_{i}$ the adsorption equilibrium constant for solute " $i$ " (dimensionless - tabulated) and $a_{i}$ the activity of component " $i$ " (dimensionless). The reader is reminded that activity represents an "effective" concentration which relies on calculations of activity coefficients. Activity coefficients are related to the molecular forces taking place in a solution. The thermodynamics of a solution mixture however depend on the intermolecular forces that operate between molecules that are dependent on the nature of the solvent(s) and solutes. For more information the reader is referred to Topping et al. (2005b) and references therein. As stated by $\mathrm{Li}$ and $\mathrm{Lu}(2001)$, the quantity $\Gamma_{i}^{w o}$ is defined as the excess of " $i$ " in a unit cross sectional area of the surface region over the moles which would be present in the bulk liquid phase containing the same number of moles of water as does the section of surface region. The superscript $w$ indicates that the dividing surface is chosen so that $\Gamma_{w}=0$. For more in-depth discussions on the dividing surface the reader is referred to sources in the literature (e.g. Sorjamaa et al., 2004). The authors claim the model is able to represent surface tensions for inorganic systems up to concentrations of $36 \mathrm{M}$ where an average absolute percentage deviation from experimental data for 45 single solutes of 0.47 was reported. In addition, it was used to predict surface tensions of $11 \mathrm{bi}$ nary and five ternary mixtures with an average absolute percentage deviation from experimental data of 1.69.

The two models presented by $\mathrm{Li}$ and Lu (2001) for mixed electrolyte solutions, which are discussed more in Sect. 3.3 have slightly different derivations. The first is based on the assumption that there is no interaction or competing adsorption between electrolytes at the interface. In other words, it assumes that the relationship defining the surface excess used in a binary system still holds in a mixture. The second model assumes that it may be preferable to consider competing adsorption between the electrolytes at the interface at higher concentrations. This seems to be important for systems with a free acid and its salt ( $\mathrm{Li}$ and $\mathrm{Lu}, 2001$ ). For all inorganic contributions to the surface tension of the mixture, this model is employed here.

\subsection{Organics}

Introducing organic compounds into the modelling framework is more complex. Whilst an in-depth discussion on the adsorption characteristics of different surfactants, and their atmospheric relevance, is beyond the scope of this study (e.g. Facchini et al., 2000; Seidl, 2000), it is well known that some organic compounds are surface active and their presence in solution can significantly affect the surface tension of cloud droplets (Facchini et al., 2000; Shulman et al., 1996; Tuckermann and Cammenga, 2004). Whereas for non-aqueous solutions the mixture surface tension in some cases can be approximated by a linear dependence, aqueous systems show pronounced non-linear characteristics. This is typical of organic-aqueous systems, in which small concentrations of the organic material may significantly affect the mixture surface tension (Poling, 2000). Here the hydrocarbon portion of the molecule behaves like a hydrophobic material and tends to be rejected from the water phase by preferentially concentrating on the surface (Poling, 2000). In such a case, the bulk concentration can be very different from the surface concentration.

\subsubsection{Binary organic systems}

Binary aqueous solutions listed in Table 1a were studied in the laboratory (see Sect. 2). Generally in order to use mixture rules to model multi-component systems one often relies upon the use of binary information to describe the behaviour of a mixture. It is unclear in the literature which predictive models, if any, are appropriate for atmospheric studies. A brief introduction and review of binary methods is given by Poling (2000). Two models presented by the authors include the techniques of Tamura et al. (1955) and Sprow and Prausnitz (1966a, b). The method of Tamura et al. (1955), which uses the Macleod-Sugden correlation as a starting point (Poling, 2000), leads to the relationship given by Eq. (3).

$$
\begin{aligned}
& \sigma_{w s}^{1 / 4}=\psi_{w} \sigma_{w}^{1 / 4}+\psi_{i} \sigma_{i}^{1 / 4} \\
& \psi_{i}=1-\psi_{w}
\end{aligned}
$$

where $\psi_{i}$ is the volume fraction of component " $i$ " in the surface layer, $\psi_{w}$ the volume fraction of water in the surface layer, $\sigma_{w s}$ is the surface tension of the mixture, $\sigma_{w}$ that of pure water and $\sigma_{i}$ the surface tension of the pure organic component " $i$ ". The latter parameter is discussed in more detail in Sect. 3.2.2, its importance for comparison 
with experimental data discussed in Sect. 4. $\psi_{w}$ is given by Eq. (5).

$$
\begin{aligned}
& \log _{10} \frac{\left(\psi_{w}\right)^{q}}{\left(1-\psi_{w}\right)}=\log _{10}\left[\frac{\left(x_{w} V_{w}\right)^{q}}{x_{i} V_{i}}\left(x_{w} V_{w}+x_{i} V_{i}\right)^{1-q}\right] \\
&+44.1 \frac{q}{T}\left[\frac{\sigma_{i} V_{i}^{2 / 3}}{q}-\sigma_{w} V_{w}^{2 / 3}\right]
\end{aligned}
$$

where $x_{w}$ is the bulk mole fraction of water, $x_{i}$ the bulk mole fraction of the organic component, $V_{w}$ the molar volume of pure water $\left(\mathrm{m}^{3} \mathrm{kmol}^{-1}\right), V_{i}$ the molar volume of the pure organic component $\left(\mathrm{m}^{3} \mathrm{kmol}^{-1}\right), T$ the temperature $(\mathrm{K})$ and $q$ is a constant that depends on the size and type of organic compound (for fatty acids and alcohols $q=$ the number of carbon atoms; for ketones $q=$ one less than the number of carbon atoms; for halogen derivatives of fatty acids $q=$ number of carbon atoms times the ratio of the molar volume of the halogen derivative to the parent fatty acid). Methods for obtaining a value for the bulk molar volume are briefly presented in Sect. 3.2.2. Tamura et al. (1955) applied the above model to 14 aqueous systems and found percentage errors of less than $10 \%$ when $q<5$ and within $20 \%$ for $q>5$.

A thermodynamic based relationship was provided originally by Sprow and Prausnitz (1966a, b). Using assumptions of equilibrium between the surface and bulk phase, and the partial molar surface area of component " $i$ " being the same as the molar surface area, leads to the relationship given by Eq. (6). By definition, the partial molar surface area will be dependent on composition and may not be equal to the molar surface area, which is calculated as an effective surface area as discussed shortly.

$\sigma_{w s}=\sigma_{i}+\frac{R^{\prime} T}{A_{i}} \ln \frac{x_{i}^{\sigma} \gamma_{i}^{\sigma}}{x_{i} \gamma_{i}}(i=1,2 \ldots . N)$

In Eq. (6) $\sigma_{i}$ is the pure component surface tension (dyne $\left.\mathrm{cm}^{-1}\right), R^{\prime}=8.314 \times 10^{7}$ dyne $\mathrm{cm}^{-1} / \mathrm{mol} \mathrm{K}, T$ the temperature $(\mathrm{K}), A_{i}$ the molar surface area of component " $i$ " in $\mathrm{cm}^{2} \mathrm{~mol}^{-1}, x_{i}$ the bulk mole fraction of component " $i$ ", $x_{i}^{\sigma}$ the surface mole fraction of component " $i$ ", $\gamma_{i}$ the activity coefficient of component " $i$ " in the bulk phase, $\gamma_{i}^{\sigma}$ the activity coefficient of component " $i$ " in the surface phase and " $N$ " is the total number of components. This has already been employed in the thermodynamic model of Ming and Russell (2002) and Topping et al. (2005b). Whilst a solution to Eq. (6) can be found using a bisection approach, Ming and Russell (2002) use a constant of proportionality between the bulk and surface phase. Suarez et al. (1989) improved on previous attempts to utilise the above relationship by using a more accurate way of calculating the different activity coefficients (UNIFAC - Fredenslund et al., 1975). However, the surface tension calculated in this way is more sensitive to the choice of " $A_{i}$ ". Goldsack and White (1983) derived an equation for " $A_{i}$ " based on the assumption that molecules were spherical and that the effective surface area is best represented by the cross sectional area as given by Eq. (7).

$A_{i}=1.021 \times 10^{8} V_{c}^{6 / 15} V_{b}^{4 / 15}$

where $V_{c}$ and $V_{b}$ are the critical and bulk molar volumes respectively $\left(\mathrm{cm}^{3} \mathrm{~mol}^{-1}\right)$. The critical molar volume is the molar volume at the critical point, which represents the point of termination on a phase equilibrium curve. Equation (7) is used in this study, a discussion of its validity beyond the scope of this paper. If the parameters used in Eq. (7) are not available experimentally then they must be calculated. In this study values for the bulk molar volume are taken from no more than two sources and are tabulated in Table 2 . One method is to calculate this value using the molecular weight and density obtained using the Yens-Wood technique (www.pirika.com). The second source is the DIPPR project archive on the Knovel database (www.knovel.com). For the critical molar volumes again two methods are used. One option is to employ the third order group contribution technique of Marrero and Gani (2001), and the other again is to use the archived DIPPR project database (www.knovel.com). Whilst Suarez et al. (1989) reported an average error of $3.5 \%$ using the above relationship for $A_{i}$ for non-aqueous mixtures, the authors also state that deviations may be $15 \%$ or more for aqueous systems which may be due to orientation effects giving rise to molar areas which differ significantly from those calculated using (7). Suarez et al. (1989) improved results considerably when deriving special area parameters from available experimental data. Unfortunately, such improvements may not be available for systems of atmospheric importance and only a small number of improved parameters have been reported (see Poling, 2000). If binary data had to be measured in the laboratory to optimise the parameter matrix for this method then it would render its predictive capability redundant. The parameters required for use in the above two models are given in Table 2.

A further option explored in this study, was the use of the framework developed by Li and Lu (2001), already discussed briefly in Sect. 3.1. In an attempt to test the ability of multi-component mixing rules for analysing mixed inorganic/organic systems, the binary data measured in the laboratory was used to fit parameters for use with the binary rule of $\mathrm{Li}$ and $\mathrm{Lu}$ (2001), hereafter referred to as the " $L i L u$ " method, given by Eq. (2). This provides a model for use at different concentrations than those used in the binary studies. It also highlights the ability of the particular model framework to capture the reduction in the solution surface tension. The LiLu model was based on the following theory. Using the Butler equation (e.g. see Hu and Lee 2004), for a single solute " $i$ ", the reversible change in surface tension at the surface can be derived as Eq. (8):

$-d \sigma_{w s}=\Gamma_{w} d \mu_{w}^{o}+\Gamma_{i} d \mu_{i}^{o}$

where $\Gamma_{w}$ and $\Gamma_{i}$ are the surface excess of water and the solute " $i$ " respectively and are based on an arbitrary dividing 
Table 2. Critical molar volume $\left(\mathrm{cm}^{3} \mathrm{~mol}^{-1}\right)$, bulk molar volume $\left(\mathrm{cm}^{3} \mathrm{~mol}^{-1}\right)$ and pure component surface tensions $\left(\right.$ dyne $\left.\mathrm{cm}^{-1}\right)$ for the separate organic components.

\begin{tabular}{|c|c|c|c|c|c|c|}
\hline \multirow[t]{3}{*}{ Compound } & \multicolumn{2}{|c|}{ Critical molar volume } & \multicolumn{2}{|c|}{ Molar volume } & \multirow{2}{*}{\multicolumn{2}{|c|}{\begin{tabular}{|l|} 
Pure component \\
surface tension
\end{tabular}}} \\
\hline & & \multirow[b]{2}{*}{$V c^{2}$} & \multirow[b]{2}{*}{$v^{\prime}$} & \multirow[b]{2}{*}{$v^{2}$} & & \\
\hline & $V c^{1}$ & & & & $\sigma^{1}$ & $\sigma^{2}$ \\
\hline Succinic acid & 311.05 & 317 & 93.2722871 & 97.4525 & 40.1589 & 61.6 \\
\hline Malonic acid & 255.25 & 258 & 77.4667707 & 73.8918 & 40.69888 & 70.5 \\
\hline Oxalic acid & 199.45 & 205 & 60.9067083 & 61.4585 & 42.26932 & 87.3 \\
\hline Glutaric acid & 366.85 & 363 & 109.966223 & 109.199 & 38.88346 & 56.1 \\
\hline Citric acid & 451.65 & 419.68 & 124.614423 & 134.762 & 60.81962 & 103.9 \\
\hline Malic acid & 325.75 & 331 & 100.574359 & 109.887 & 37.5103 & 86.2 \\
\hline Maleic acid & 315.59 & 297 & 87.8936178 & 90.835 & 40.69888 & 67.6 \\
\hline Adipic acid & 422.65 & 400 & 149.017538 & 133.942 & 45.50509 & 52.4 \\
\hline Fulvic acid & 1818.29 & $\ldots$ & 432.2 & -- & $5.112449^{*}$ & 102.9 \\
\hline Levoglucosan & 431.14 & $\ldots$ & 127.484198 & ... & 22.7104 & -.. \\
\hline Pinic acid & 523.75 & $-\ldots$ & 180.024576 & --- & 23.74566 & -- \\
\hline & & & & & & \\
\hline & & & & & & \\
\hline \multicolumn{7}{|c|}{$\mathrm{V}_{\mathrm{c}}{ }^{1}$-Calculated using the third order group contribution technique of Marrero and Gani (2001) } \\
\hline \multicolumn{7}{|c|}{$\mathrm{V}_{\mathrm{c}}{ }^{2}-\mathrm{T}$ aken from the DIPPR online database (www knovel.com) } \\
\hline \multicolumn{7}{|c|}{$\mathrm{V}^{1}$-Calculated using the Yens-Wood density value (wnww pirika.com) } \\
\hline \multicolumn{7}{|c|}{$\mathrm{V}^{2}$ - Taken from the DIPPR online database (www knovel.com) } \\
\hline \multicolumn{7}{|c|}{$\sigma^{1}-$ Calculated using the Macleod-Sugden method and Yens-Wood density (www pirika.com) } \\
\hline \multicolumn{5}{|c|}{$\sigma^{2}$-Calculated using Chemsketch Version 5.0 (www acdlabs com) } & & \\
\hline
\end{tabular}

surface ( $\mathrm{Li}$ and $\mathrm{Lu}, 2001$ ). Rewriting in terms of activities, using the Gibbs dividing surface and choosing $\Gamma_{w}=0$, Eq. (8) reduces to Eq. (9):

$-d \sigma_{w s}=\Gamma_{i}^{w} R T d \ln \left(a_{i}\right)$

where the superscript $w$ on $\Gamma$ indicates that the diving surface is chosen so that $\Gamma_{w}=0$. Next an assumption is made that the solute adsorption on the surface of solution is considered to be behaving in the same manner as that in the Langmuir gas - solid adsorption ( $\mathrm{Li}$ and $\mathrm{Lu}, 2001$ ). The validity of this assumption is not analysed here, rather the ability of the finalised model to fit to the binary data reviewed. Following this assumption an expression for $\Gamma_{i}^{w}$ can be derived as:

$\Gamma_{i}^{w}=\Gamma_{i}^{w o} \frac{K_{i} a_{i}}{1+K_{i} a_{i}}$

where $\Gamma_{i}^{w o}$ is the saturated surface excess of solute " $i$ ", $K_{i}$ the adsorption equilibrium constant and $a_{i}$ the activity of solute " $i$ ". Substituting Eq. (10) into Eq. (9) and integrating leads to Eq. (11) for the surface tension:

$\sigma_{w s}=\sigma_{w}+R T \Gamma_{i}^{w o} \ln \frac{1}{1+K_{i} a_{i}}$

where $\sigma_{w}$ is the surface tension of pure water at the desired temperature. In this study, activity coefficients were calculated using the UNIFAC activity coefficient model on a mole fraction basis. These calculations were made using the revised parameters of Peng et al. (2001). To fit the two parameters for this surface tension model the large scale optimisation algorithm of the MATLAB (R) 6.1 (www.mathworks. com) software package was used. Calculating the jacobian matrix using a finite difference technique, the initial starting points were chosen using a random number generator extracting numbers from a normal distribution over 1000 iterations. The parameters derived from the fitting procedure are shown in Table 1a along with the range of validity.

\subsubsection{Pure component parameters}

As shown in previous sections, predictive binary methods are available which often rely on the use of pure component surface tension data. For most organic liquids, this ranges between 25 and 40 dyne $\mathrm{cm}^{-1}$ (Reinhard and Drefahl, 1999). Unfortunately, for surface tension modelling there is a lack of such data, some organic components of atmospheric importance being solid at room temperature. As noted by Poling (2000), essentially all useful estimation techniques for the surface tension of a liquid are empirical. Critical evaluations of experimental surface tensions for pure compounds are provided in the literature (e.g. Jasper, 1972; Riddick et al., 1986). However, again the scope of the datasets is such that predictive schemes are likely to be required for species of atmospheric significance since they may be solid at room temperature. In this instance, the pure surface tensions with respect to the super-cooled liquid state must be calculated (Gaman et al., 2004). In the literature there are techniques presented which depend on the nature of the organic compound in question. Poling (2000) reviews some of these methods for both polar and non-polar molecules. For 
hydrocarbon and polar molecules (Perry and Green, 1997) the Macleod-Sugden correlation shown in Eq. (12) is often employed (Poling, 2000):

$\sigma_{O}^{1 / 4}=[P]\left(\rho_{L}-\rho_{V}\right)$

where $\sigma_{O}$ is the pure component surface tension $(\mathrm{mN} / \mathrm{m})$, $[\mathrm{P}]$ is called the Sugden parachor, $\rho_{L}$ the pure component liquid density and $\rho_{V}$ the vapour density $\left(\mathrm{kmol} / \mathrm{m}^{3}\right)$. Usually the vapour density is neglected for systems under low pressure and one has to calculate the liquid density if experimental data does not exist. This is discussed in more detail below. Quayle (1953) suggested an additive scheme to correlate $[\mathrm{P}]$ with structure using experimental data for surface tension and density for many compounds. The noted "good performance" and simplicity have made the above scheme a very popular method (Escobedo and Mansoori, 1996). Multi-parametric correlations between $\sigma_{O}$ and physicochemical and molecular properties are provided in the literature (Reinhard and Drefahl, 1999). However, these seem to be restricted to alkanes (Needham et al., 1988), hydrocarbons, halogenated hydrocarbons, alkanols, ethers, ketones and esters (Stanton and Jurs, 1990). It is more common, and considerably easier, to rely on a group contribution approach. In this study, the parachor is calculated using the Advanced Chemistry Development Inc. (ACD/Labs) software Chemsketch v5.0 (ChemSketch, 2003) by inputting the appropriate structure which can be found in the NIST chemistry web book (www.nist.gov). Other group contribution techniques are discussed briefly by Reinhard and Drefahl (1999) and are not reviewed here.

As an alternative to the Macleod-Sugden correlation, temperature dependent pure component surface tensions are recorded in texts such as the Knovel DIPPR Project 801database (www.knovel.com). For example, using this database, the temperature dependence of the pure component surface tension for glutaric acid is given as:

$\sigma_{o}=0.078398[1-\operatorname{Tr}]^{(1.105)}$

where $\sigma_{o}$ is given in $(\mathrm{N} / \mathrm{m}), \operatorname{Tr}$ is the reduced temperature $\left(T / T_{c}\right)$ and $T_{c}$ is the critical temperature. However, the minimum temperature at which the fit is valid is given as $370.05 \mathrm{~K}$, which is noted as the melting point/triple point. It is difficult to judge how valid Eq. (13) is at lower temperatures. Indeed, at $298.15 \mathrm{~K}$, using the critical temperature of $807 \mathrm{~K}$, the above formulation gives a surface tension of $0.0471 \mathrm{Nm}^{-1}$. However, use of ACDlabs Chemsketch 5.0, another direct alternative for calculating $\sigma_{o}$, gives a value of $0.0561 \mathrm{Nm}^{-1}$, a difference of $19 \%$.

Escobedo and Mansoori (1996) noted various shortcomings associated with the use of Eq. (12). These include the fact that the parachor $[\mathrm{P}]$ is actually a temperature-dependent parameter whose functional form with temperature is not known. However, results appear to be species specific since it has been noted to work very well for many substances and over a wide range of temperature (Escobedo and Mansoori, 1996). The other shortcomings were related to the empirical nature of the parachor, thus creating difficulty in deriving a more accurate expression for it, and the fact that the deviation between measured surface tension and that predicted increases as the complexity of the molecular structure of the fluid under consideration increases (Escobedo and Mansoori, 1996).

If one obtains a value for the parachor independently then in addition one may need to calculate the liquid density. Again, since some organics of atmospheric interest may be solid at room temperatures then this requires the use of predictive techniques. Following Gaman et al. (2004) the pure sub-cooled acid densities can be calculated as a function of temperature using the Yens-Wood method (www.pirika.com). The calculated pure surface tension is very sensitive to the value of the liquid density used. For example, the ACDlabs Chemsketch 5.0 and the Yens-Wood technique give density values of 1.408 and $1.266 \mathrm{gcm}^{-3}$ for Succinic acid respectively. Similarly for Glutaric acid, the values are 1.32 and $1.20 \mathrm{gcm}^{-3}$ respectively. Whilst they differ by around $10 \%$ this translates to a difference in the pure surface tension of 21.4 and $17.2 \mathrm{dyne}^{-1}$ for succinic and glutaric acid

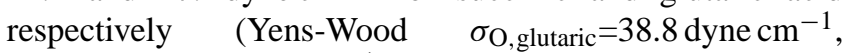
$\sigma_{\mathrm{O} \text {, succinic }}=40.1 \mathrm{dyne}^{-1}$; $\quad$ ACDLabs $\sigma_{\mathrm{O} \text {, glutaric }}=56.1$ dyne $\mathrm{cm}^{-1}, \quad \sigma_{\mathrm{O} \text {, succinic }}=61.6 \mathrm{dyne} \mathrm{cm}^{-1}$ ). A larger difference was found for oxalic acid where the ACDlabs calculation leads to a surface tension greater than that of pure water. The Yens-Wood method however leads to a value of around $56.1 \mathrm{dyne} \mathrm{cm}^{-1}$. In the following body of text, the sensitivity to the choice of $\sigma_{O}$ is explored on comparison with binary data. The range of values found for compounds analysed in this study are given in Table 2.

\subsection{Multi-component modelling techniques}

It is now pertinent to analyse ways of predicting the surface tension for multi-component systems. These include mixed organic and mixed inorganic/organic systems. Before comparisons with experimental data are made, a review of the models and combinations thereof are given. Table 4 lists all combinations used. The simplest approach is to add the contributions from either separate compounds or the inorganic and organic fractions. In this study, when comparing with mixed inorganic/organic systems, the ability of a simple additive approach is analysed. More specifically, the deviations from the surface tension of pure water calculated by separate inorganic and organic schemes are simply added:

$\sigma_{w s}=\sigma_{w}+\Delta \sigma_{o r g}+\Delta \sigma_{i n o r g}$

where $\Delta \sigma_{\text {org }}$ and $\Delta \sigma_{\text {inorg }}$ are the deviations from the surface tension of pure water caused by the organic and inorganic components respectively. However there are other options 
for coupling the organic and inorganic components based on models provided in the literature.

The techniques for multi-component inorganic mixtures were discussed briefly in Sect. 3.1. For all multi-component inorganic calculations, the model of $\mathrm{Li}$ and $\mathrm{Lu}$ (2001) is employed. Theoretically this framework can be applied to mixed organic and mixed inorganic/organic systems provided the appropriate parameters are available. Such parameters have already been derived and are discussed in Sect. 3.2.1. However, for multi-component systems, Li and $\mathrm{Lu}$ (2001) proposed two methods. The first method was to propose that the adsorption behaviour of solute " $i$ " still followed the Langmuir gas-solid adsorption model, applied previously, in a mixed solution. Thus it assumes that there is no interaction, nor competing adsorption, from the other solutes. In other words, it assumes that the relationship defining the surface excess used in a binary system still holds in a mixture. Combining with the Gibbs dividing surface represented by $\Gamma_{w}=0$ this gives Eq. (15):

$\sigma_{w s}=\sigma_{w}+R T \sum_{i=1}^{K} \Gamma_{i}^{w o} \ln \left(\frac{1}{1+K_{i} a_{i}}\right)$

However, the authors also note how it may be possible to consider the interactions in the interface at higher concentrations. By assuming the adsorption rate of a specie " $i$ " in the mixed layer is equal to the de-sorption rate leads to a new definition for the saturated surface excess. This yields another expression for the mixed solution surface tension:

$\sigma_{w s}=\sigma_{w}+R T \sum_{i=1}^{K} \Gamma_{i}^{w o} \ln \left(1-\frac{K_{i} a_{i}}{1+\sum_{j} K_{j} a_{j}}\right)$

There are various options for calculating activities of the solutes. One option is to assume semi ideality, using the total water content to derive appropriate concentrations for each binary system. Semi ideality assumes that there is no interaction between solutes in a mixture, only that between each solute and the solvent. For example, when calculating water contents one would simply add the water from each binary system. For calculating activity coefficients one would assume that each compound is present on its own at the concentration defined using the total water content. If one assumes ideality then the activity of each compound is simply represented by its concentration in the mixture and using activity coefficients of unity. Another option is to explicitly calculate the activities for the mixed fraction, be it inorganic or organic. Of course, a generic method for coupling the inorganic and organic fractions, thus treating the influence of inorganic-organic interactions, is currently not possible (Clegg and Seinfeld, 2006; Clegg et al., 2001) so only the effect of the inorganic ions is taken into account when calculating inorganic activities and vice versa for the organic fraction. The different combinations employed on compar- ison with experimental data are clearly identified in Sect. 4 onward.

Now attention is given solely to mixed organic systems. Mixing rules are used to calculate various properties, such as water content and solution density. They can nominally be distinguished from other techniques, such as full predictive frameworks, as they employ, in one way or another, data from binary mixtures to describe the properties of multicomponent systems. As discussed by Fainerman and Miller (2001), most authors have proposed procedures or models to predict the adsorption behaviour for a surfactant mixture from the known surface characteristics of the single compound. This naturally requires detailed experimental studies of surface tensions of individual solutions, and often, additional parameters that account for the mutual influence of solutes. This imposes certain restrictions on the capability of such approaches (Fainerman et al., 2001). Whilst theoretical predictive frameworks exist, the ability to capture the varying surface tension is hindered by a neglect of important processes or parameters which can define the behaviour of a mixed surfactant system (Fainerman et al., 2001). Following this, Fainerman et al. (2002) derived a general but simple approximate expression for the surface tension of a surfactant mixture that allows estimation of the characteristics of a mixed solution, without any detailed analysis of the behaviour of the individual solutions and/or any account for specific interactions between the mixed species. It has been shown that it is possible to predict the surface tension of a mixed solution of two (or even " $n$ ") surfactants of different natures from the surface tensions for the individual solutions using this scheme. This is discussed in more detail as follows.

Equations of state for surface layers, adsorption isotherms and surface tension isotherms can be derived by equating the expressions for the chemical potentials at the surface and those in the solution bulk. Fainerman et al. (2002) derived generalised expressions from which many known isotherms for non-ionic surfactants can be obtained. For example, by employing a surface layer model in which the molar surface area of the solvent is equal to that of the solute, and assuming that the surface and bulk phase is ideal, then one can derive the von Szyskowski equation and Langmuir isotherm (Fainerman et al., 2002). Modifications of the generalised expressions resulted in frameworks that were designed for specific systems. For example, variations resulted from using regular solution theory to account for terms representing intermolecular interactions and employing different representations of the molar surface areas. However, Fainerman and Miller (2001) note that applications of such complex equations would require adsorption characteristics of individual surfactant solutions. Also, since coupling of intermolecular interactions is uncertain, these formulations could not be trusted to ensure a correct description for any surfactant mixture. Thus, an approximate approach was developed. Considering an ideal mixture of homologues, the "generalised" 
Szyskowski equation was derived. This was then further generalised to a system of $\mathrm{n}$ components to give the following equation of state, hereafter referred to as the $F M$ mixing rule:

$\exp \bar{\prod}_{\mathrm{sol}}=\sum_{i=1}^{n} \exp {\overline{\prod_{i}}}+1-n$

where

$\bar{\prod}_{\mathrm{sol}}=\frac{\prod_{\mathrm{sol}} \omega}{R T}$

and

$\bar{\prod}_{i}=\frac{\prod_{i} \omega}{R T}$

where $\omega$ is the average molar surface area of the solution, $\prod_{\text {sol }}$ and $\prod_{i}$ are the surface pressures of the solution and solute given by:

$\prod_{s o l}=\sigma_{w s}-\sigma_{\mathrm{sol}}$
$\prod_{i}=\sigma_{w s}^{i}-\sigma_{\mathrm{sol}}$

where $\sigma_{w s}$ is the surface tension of the solution, $\sigma_{\text {sol }}$ the surface tension of the solvent and $\sigma_{w s}^{i}$ the surface tension of the binary system " $i$ ". If the partial molar areas of the components are different then the average value for the mixture can be calculated from Eq. (22) (Fainerman et al., 2002):

$\omega=\frac{\sum_{i} \omega_{i} \prod_{i}}{\sum_{i} \prod_{i}}$

where $\omega_{i}$ is the molar area of component " $i$ ". The model is very general, as it gives a tool how to merge the properties of different compounds into the interfacial behaviour of the mixture. In the above framework, for each component a different model can be advantageous. To import this particular behaviour into the behaviour of the mixture you only need to calculate the dimensionless surface pressure at the given conditions (Reinhard Miller private communication ${ }^{1}$ ). Fainerman et al. (2001) compared the above formulation with experimental data and found it to be valid for several different surfactant systems, even those containing components with large differences in molar surface areas and other adsorption parameters. Fainerman et al. (2001) state this validity is ascribed to the fact that many particular features of adsorption process in mixed components (surface layer non-ideality, capability to reorient at the surface layer etc.) are accounted for 'automatically' because the surface tensions of the individual solutions are used. It does not however take into account the specific interaction between the mixed molecules and it is of

\footnotetext{
${ }^{1}$ Dr. habil. Reinhard Miller, Max-Planck-Institut fuer Kolloidund Grenzflaechenforschung, 24 May 2006
}

course an approximation. However, its simplicity and ease of use makes it useful for estimating behaviour for many systems, its applicability for atmospheric compounds analysed here. In this study, parameters derived from fitting to binary data are used with Eq. (2) for representing the binary systems at varying concentrations as discussed in Sect. 3.2.2.

Interestingly, when Eq. (2) is used to describe the surface tension of binary systems in the $F M$ mixing rule then predictions for multicomponent organic aqueous systems are identical to the "full" LiLu thermodynamic model. Note that the "full" $L i L u$ model refers to the $L i L u$ model framework applicable to multicomponent systems (Eq. 15). This is discussed more in Sect. 4 To explain this similarity, consider the following discussion. For a ternary system, the $F M$ mixing rule reduces to:

$$
\exp _{\bar{\Pi}}=\exp {\overline{\prod_{1}}}+\exp {\overline{\Pi_{2}}}^{-1}
$$

Substituting Eqs. (18) and (19) into Eq. (23) and taking the logarithm on the resulting expression, this further simplifies to:

$\frac{\prod \omega_{w s}}{R T}=\frac{\prod_{1} \omega_{1}}{R T}+\frac{\prod_{2} \omega_{2}}{R T}$

Now replacing the individual molar surface areas with the average value calculated using Eq. (22) and introducing the relevant surface tensions gives:

$$
\begin{aligned}
& \frac{\left(\sigma_{\mathrm{sol}}-\sigma_{w s}\right) \omega}{R T}=\frac{\left(\sigma_{\mathrm{sol}}-\sigma_{w s}^{1}\right) \omega}{R T}+\frac{\left(\sigma_{\mathrm{sol}}-\sigma_{w s}^{2}\right) \omega}{R T} \\
& \frac{\sigma_{w s} \omega}{R T}=\frac{\sigma_{w s}^{1} \omega}{R T}+\frac{\sigma_{w s}^{2} \omega}{R T}-\frac{\sigma_{\mathrm{sol}} \omega}{R T}
\end{aligned}
$$

Inserting Eq. (13) to represent the binary surface tension:

$$
\begin{aligned}
& \frac{\sigma_{w s} \omega}{R T}=\left(\sigma_{\mathrm{sol}}+R T \Gamma_{1}^{0} \ln \left(\frac{1}{1+K_{1} a_{1}}\right)\right) \frac{\omega}{R T} \\
& +\left(\sigma_{\mathrm{sol}}+R T \Gamma_{2}^{0} \ln \left(\frac{1}{1+K_{2} a_{2}}\right)\right) \frac{\omega}{R T}-\frac{\sigma_{\mathrm{sol}} \omega}{R T} \\
& \frac{\sigma_{w s} \omega}{R T}=\frac{\sigma_{\mathrm{sol}} \omega}{R T}+R T \Gamma_{1}^{0} \ln \left(\frac{1}{1+K_{1} a_{1}}\right) \frac{\omega}{R T} \\
& +R T \Gamma_{2}^{0} \ln \left(\frac{1}{1+K_{2} a_{2}}\right) \frac{\omega}{R T} \\
& \frac{\sigma_{w s} \omega}{R T}=\frac{\sigma_{\mathrm{sol}} \omega}{R T}+R T\left(\frac{\omega}{R T}\right) \sum_{i} \Gamma_{i}^{0} \ln \left(\frac{1}{1+K_{i} a_{i}}\right)
\end{aligned}
$$

Rewriting the surface tension of the solvent to be that of pure water:

$$
\sigma_{w s}=\sigma_{w}+R T \sum_{i} \Gamma_{i}^{0} \ln \left(\frac{1}{1+K_{i} a_{i}}\right)
$$


Table 3a. Average percentage deviations on comparing model results with experimental data for malonic, maleic and malic acid using two predictive binary techniques (Tamura/Suarez - described in the text) and one model fit to the data ( $L i L u$ - described in the text).

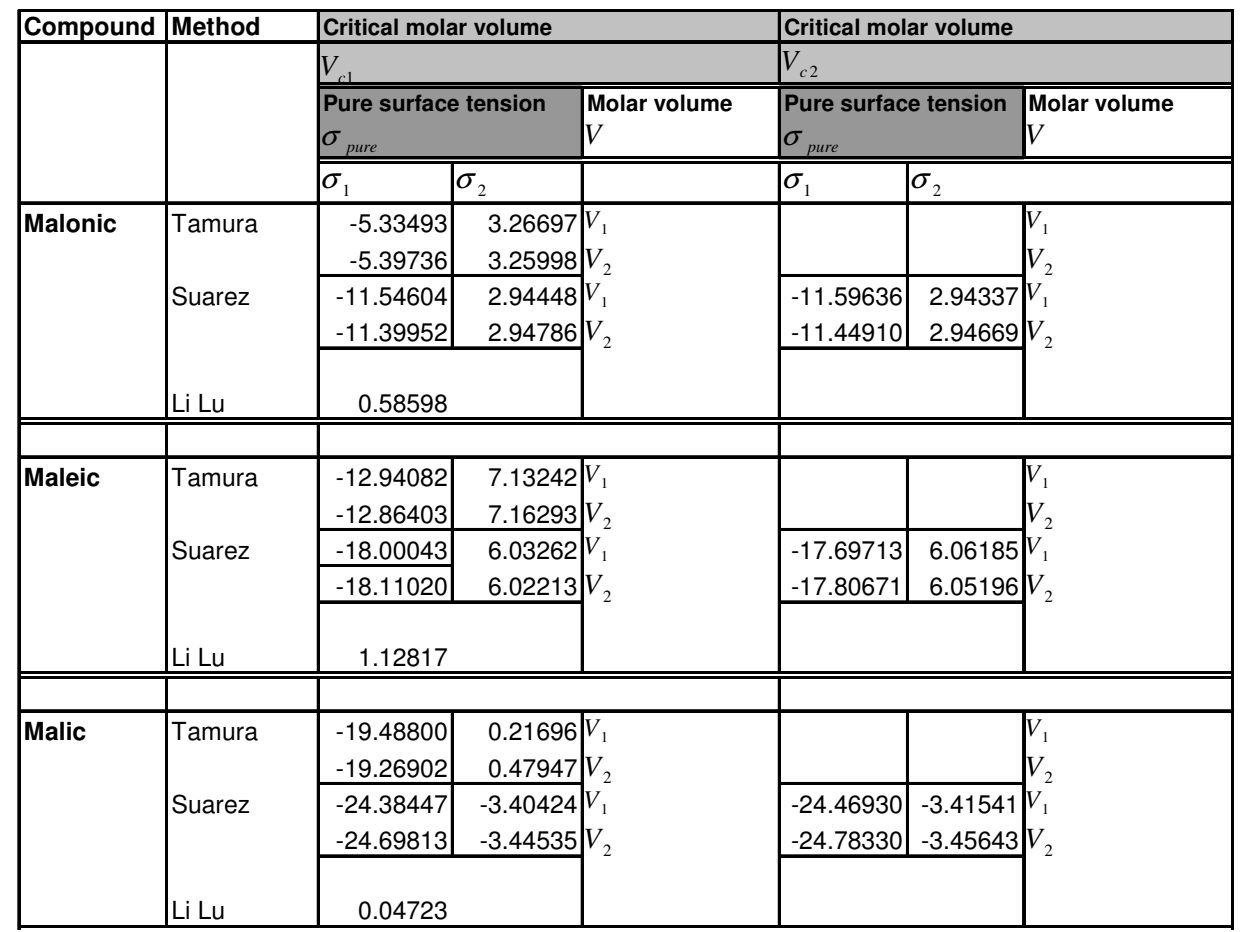

Table 3b. Average percentage deviations on comparing model results with experimental data for glutaric, citric and pinonic acid using two predictive binary techniques (Tamura/Suarez - described in the text) and one model fit to the data ( $L i L u$ - described in the text).

\begin{tabular}{|c|c|c|c|c|c|c|c|}
\hline \multirow[t]{4}{*}{ Compound } & \multirow[t]{4}{*}{ Method } & \multicolumn{3}{|c|}{ Critical molar volume } & \multicolumn{3}{|c|}{ Critical molar volume } \\
\hline & & \multicolumn{3}{|l|}{$V_{c 1}$} & \multicolumn{3}{|l|}{$V_{c 2}$} \\
\hline & & \multicolumn{2}{|c|}{\begin{tabular}{|l} 
Pure surface tension \\
$\sigma_{\text {pure }}$ \\
\end{tabular}} & \multirow[t]{2}{*}{$\begin{array}{l}\text { Molar volume } \\
V\end{array}$} & \multicolumn{2}{|c|}{\begin{tabular}{|l} 
Pure surface tension \\
$\sigma_{\text {pure }}$ \\
\end{tabular}} & \multirow[t]{2}{*}{$\begin{array}{l}\text { Molar volume } \\
V\end{array}$} \\
\hline & & $\overline{\sigma_{1}}$ & $\overline{\sigma_{2}}$ & & $\sigma_{1}$ & $\sigma_{2}$ & \\
\hline \multirow[t]{5}{*}{ Glutaric } & \multirow[t]{2}{*}{ Tamura } & -12.25911 & 2.42443 & \multirow{5}{*}{$\begin{array}{l}V_{1} \\
V_{2} \\
V_{1} \\
V_{2}\end{array}$} & -12.25911 & 2.42443 & \multirow{5}{*}{$\begin{array}{l}V_{1} \\
V_{2} \\
V_{1} \\
V_{2}\end{array}$} \\
\hline & & -12.28212 & 2.40479 & & -12.28212 & 2.40479 & \\
\hline & \multirow[t]{2}{*}{ Suarez } & -14.74051 & 1.06126 & & -14.67185 & 1.08246 & \\
\hline & & -14.71012 & 1.07066 & & -14.64150 & 1.09182 & \\
\hline & Li Lu & \multicolumn{2}{|l|}{0.64695} & & & & \\
\hline \multirow{6}{*}{ Citric } & \multirow{5}{*}{$\begin{array}{l}\text { Tamura } \\
\text { Suarez }\end{array}$} & & & \multirow{6}{*}{$\begin{array}{l}V_{1} \\
V_{2} \\
V_{1} \\
V_{2}\end{array}$} & & & \multirow{6}{*}{$\begin{array}{l}V_{1} \\
V_{2} \\
V_{1} \\
V_{2}\end{array}$} \\
\hline & & -0.63722 & -0.39153 & & -0.63722 & -0.39153 & \\
\hline & & -0.44750 & 0.24390 & & -0.44750 & 0.24390 & \\
\hline & & \multirow{2}{*}{\multicolumn{2}{|c|}{$\begin{array}{l}-0.62068 \\
-0.68793\end{array}$}} & & -0.52901 & & \\
\hline & & & & & -0.59480 & & \\
\hline & Li Lu & \multicolumn{2}{|l|}{-0.39365} & & & & \\
\hline & & & & & & & \\
\hline \multirow[t]{3}{*}{ Pinonic } & \multirow{2}{*}{$\begin{array}{l}\text { Tamura } \\
\text { Suarez }\end{array}$} & -19.77190 & \begin{tabular}{|l|}
-14.58075 \\
\end{tabular} & \multirow{3}{*}{$\begin{array}{l}V_{1} \\
V_{1}\end{array}$} & & & \\
\hline & & -26.50751 & -20.55940 & & & & \\
\hline & Li Lu & \multicolumn{2}{|l|}{-0.05814} & & & & \\
\hline
\end{tabular}


Table 3c. Average percentage deviations on comparing model results with experimental data for oxalic, succinic and adipic acid using two predictive binary techniques (Tamura/Suarez - described in the text) and one model fit to the data ( $L i L u$ - described in the text).

\begin{tabular}{|c|c|c|c|c|c|c|c|}
\hline \multirow[t]{4}{*}{ Compound } & \multirow[t]{4}{*}{ Method } & \multicolumn{3}{|c|}{ Critical molar volume } & \multirow{2}{*}{\multicolumn{3}{|c|}{\begin{tabular}{|l} 
Critical molar volume \\
$V_{c 2}$
\end{tabular}}} \\
\hline & & \multicolumn{3}{|l|}{$V_{c 1}$} & & & \\
\hline & & \multicolumn{2}{|c|}{$\begin{array}{l}\text { Pure surface tension } \\
\sigma_{\text {pure }}\end{array}$} & \multirow[t]{2}{*}{$\begin{array}{l}\text { Molar volume } \\
V\end{array}$} & \multicolumn{2}{|c|}{\begin{tabular}{|l|} 
Pure surface tension \\
$\sigma_{\text {pure }}$
\end{tabular}} & \multirow[t]{2}{*}{$\begin{array}{l}\text { Molar volume } \\
V\end{array}$} \\
\hline & & $\overline{\sigma_{1}}$ & $\sigma_{2}$ & & $\sigma_{1}$ & $\sigma_{2}$ & \\
\hline \multirow[t]{5}{*}{ Oxalic } & Tamura & -0.85234 & \multirow{5}{*}{\begin{tabular}{|r|r|} 
& 1.17606 \\
1.17760 \\
-0.36012 \\
-0.36215 \\
\end{tabular}} & \multirow{5}{*}{$\begin{array}{l}V_{1} \\
V_{2} \\
V_{1} \\
V_{2}\end{array}$} & -0.85234 & 1.17606 & \multirow{5}{*}{$\begin{array}{l}V_{1} \\
V_{2} \\
V_{1} \\
V_{2}\end{array}$} \\
\hline & \multirow{3}{*}{ Suarez } & -0.84549 & & & -0.84549 & 1.17760 & \\
\hline & & -8.68333 & & & -8.79960 & -0.37031 & \\
\hline & & -8.70870 & & & -8.82524 & -0.37259 & \\
\hline & Li Lu & 0.82081 & & & & & \\
\hline \multirow[t]{5}{*}{ Succinic } & \multirow{4}{*}{$\begin{array}{l}\text { Tamura } \\
\text { Suarez }\end{array}$} & -6.22740 & \multirow{5}{*}{\begin{tabular}{|r|}
0.51426 \\
0.56484 \\
-0.22524 \\
-0.24956 \\
\end{tabular}} & & -6.22740 & 0.51426 & \multirow{5}{*}{$\begin{array}{l}V_{1} \\
V_{2} \\
V_{1} \\
V_{2}\end{array}$} \\
\hline & & -6.10191 & & $V_{2}$ & -6.10191 & 0.56484 & \\
\hline & & -9.71645 & & & -9.82338 & -0.24050 & \\
\hline & & -9.88171 & & $V_{2}$ & -9.98978 & -0.26485 & \\
\hline & Li Lu & 0.53459 & & & & & \\
\hline \multirow[t]{5}{*}{ Adipic } & \multirow[t]{2}{*}{ Tamura } & -10.38570 & -1.63792 & $V_{1}$ & -10.38570 & -1.63792 & \multirow{5}{*}{$\begin{array}{l}V_{1} \\
V_{2} \\
V_{1} \\
V_{2}\end{array}$} \\
\hline & & \begin{tabular}{|l|}
-11.01996 \\
\end{tabular} & -2.18231 & $V_{2}$ & -11.01996 & -2.18231 & \\
\hline & \multirow[t]{3}{*}{ Suarez } & -10.15484 & -0.55326 & $V_{1}$ & -9.68556 & -0.35735 & \\
\hline & & -9.54974 & -0.30145 & $V_{2}$ & -9.08567 & -0.11297 & \\
\hline & & -0.01682 & & & & & \\
\hline
\end{tabular}

Table 3d. Average percentage deviations on comparing model results with experimental data for levoglucosan and Suwannee river fulvic acid using two predictive binary techniques (Tamura/Suarez - described in the text) and one model fit to the data ( $L i L u$ - described in the text).

\begin{tabular}{|c|c|c|c|c|}
\hline \multirow{4}{*}{ Compound } & \multirow[t]{4}{*}{ Method } & \multicolumn{3}{|c|}{ Critical molar volume } \\
\hline & & \multicolumn{3}{|l|}{$V_{c 1}$} \\
\hline & & \multicolumn{2}{|c|}{$\begin{array}{l}\text { Pure surface tension } \\
\sigma_{\text {pure }} \\
\end{array}$} & \multirow[t]{2}{*}{$\begin{array}{l}\text { Molar volume } \\
V\end{array}$} \\
\hline & & $\sigma_{1}$ & $\sigma_{2}$ & \\
\hline \multirow[t]{3}{*}{ Levoglucosan } & Tamura & -48.66224 & & \multirow{3}{*}{$\begin{array}{l}V_{1} \\
V_{1}\end{array}$} \\
\hline & Suarez & \begin{tabular}{|l}
-47.85532 \\
\end{tabular} & & \\
\hline & Li Lu & \multicolumn{2}{|l|}{-0.314542} & \\
\hline \multirow[t]{3}{*}{ Fulvic } & \multirow{3}{*}{$\begin{array}{l}\text { Tamura } \\
\text { Suarez }\end{array}$} & -82.1311 & -12.94216 & \multirow{3}{*}{$\begin{array}{l}V_{1} \\
V_{1}\end{array}$} \\
\hline & & \begin{tabular}{|l|}
-80.21784 \\
\end{tabular} & & \\
\hline & & \multicolumn{2}{|l|}{0.191486} & \\
\hline
\end{tabular}

This is identical to Eq. (15). However, this is not to be confused with the flexibility of the $F M$ approach which can employ any surface tension model to describe the binary system. Whether other binary models are required than the one employed here for compounds of atmospheric interest would form an interesting focus for future studies. To arrive back at Eq. (23) from Eq. (30) would require the same assumptions regarding the adsorption isotherms and molar surface areas.

\section{Results and discussion}

In the following section the applicability of the models discussed are analysed for binary, ternary and multi-component mixtures studied in the laboratory (see Sect. 2). 

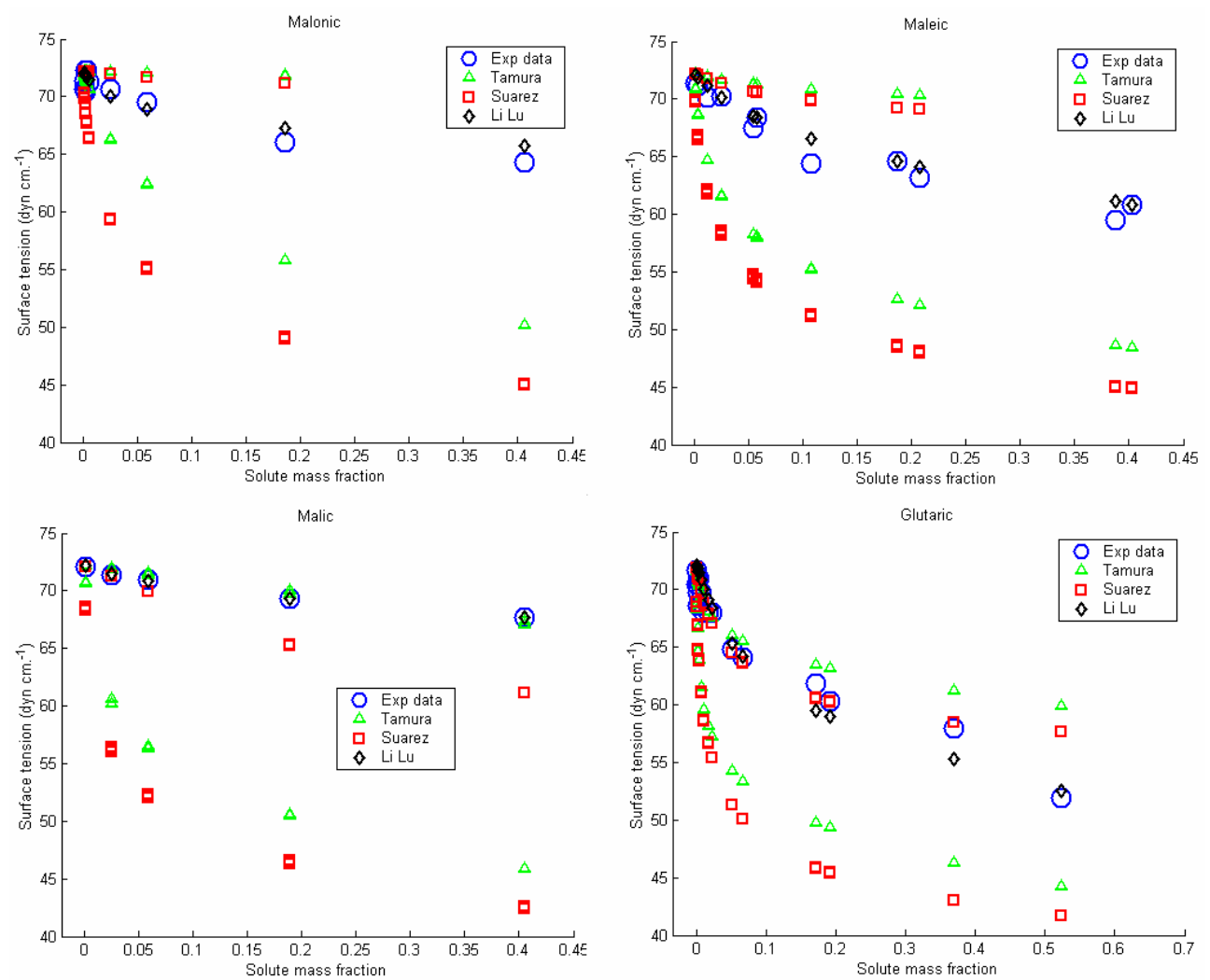

Fig. 1a. Measured surface tension for Malonic, Maleic, Malic and Glutaric acid, ("Exp data"), versus predictions from the Tamura mixing rule "Tamura" and Suarez thermodynamic method "Suarez". Also shown are predictions from the LiLu thermodynamic model fit to the binary data (" $L i L u$ "). All models are described in the text. Two sets of data represented by the same markers result from essentially two choices of pure component surface tensions within the predictive models.

\subsection{Binary organic systems}

The measured binary data is shown in Figs. 1a-c. The ability of the binary methods to reproduce the measured surface tension data is highlighted in the same figures. For the two predictive models, labelled as 'Tamura' and "Suarez", a range of values is given (Eq. (3) and Eq. (6) respectively). These apparently two distinct bands of values are essentially caused by two different values for the pure component surface tension, though the other relevant parameters were also varied. Tables $3 \mathrm{a}-\mathrm{c}$ also show the average percentage deviation (APD) on comparing the models with experimental data. Results clearly indicate that the fitted $L i L u$ model can capture the variability of the measured data very well, producing the lowest APD for all compounds studied. The performance of the other models seems to vary with compound and choice of model parameters. The Suarez thermodynamic model predictions vary with both choice of critical molar volume and bulk molar volume, whereas the Tamura mixing rule depends only on the bulk molar volume. Similarly, both methods vary with choice of pure component surface tension. Indeed, it is the latter dependence which can produce largest deviations. For example, for malic acid the difference in the pure compo- nent surface tension leads to deviations of -24.4 to $-3.40 \%$ for the Suarez method and -19.5 to $0.217 \%$ for the Tamura mixing rule. In comparison, the change caused by use of different values for the molar surface area (bulk and critical molar volumes) does not seem to produce significant difference. However, generally the two sources for these parameters give similar values. The pure component surface tension of fulvic acid is such that for one particular value $\left(102.9\right.$ dyne $\left.\mathrm{cm}^{-1}\right)$ there is no solution to the Suarez thermodynamic model using the UNIFAC activity coefficient model. As such, this technique drastically over-predicts the reduction in surface tension for the binary fulvic acid/water system by using the alternative pure component surface tension of 5.1 dyne $\mathrm{cm}^{-1}$.

The above analysis shows that our ability to predict the surface tension of even binary systems using entirely predictive frameworks is in question. Whilst a model framework was found to improve predictions considerably by fitting the appropriate parameters, it is now important to extend this analysis to multi-component mixtures. 

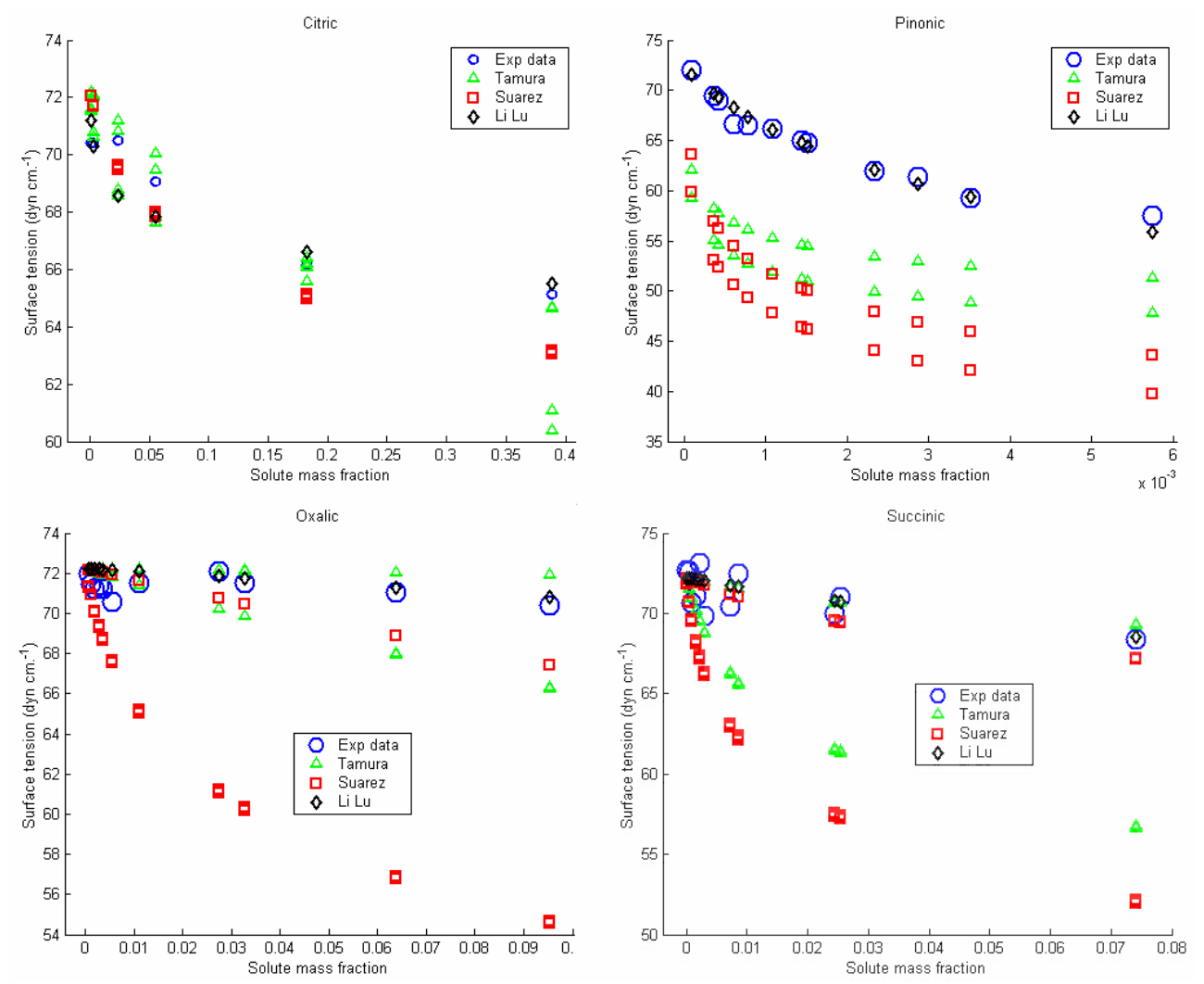

Fig. 1b. Measured surface tension for Citric, Pinonic, Oxalic and Succinic acid ("Exp data"), versus predictions from the Tamura mixing rule "Tamura" and Suarez thermodynamic method "Suarez". Also shown are predictions from the LiLu thermodynamic model fit to the binary data ("LiLu"). All models are described in the text. Two sets of data represented by the same markers result from essentially two choices of pure component surface tensions within the predictive models.

\subsection{Ternary Inorganic/Organic systems}

Three ternary systems (denoted "Tern1", "Tern2" and "Tern3") composed of Pinonic acid:( $\left.\mathrm{NH}_{4}\right)_{2} \mathrm{SO}_{4}$, Succinic acid:( $\left.\mathrm{NH}_{4}\right)_{2} \mathrm{SO}_{4}$ and Oxalic acid: $\mathrm{NaCl}$ at mass ratios of 1:1, 1:1 and 55:45 respectively were studied in the laboratory and are shown in Figs. 2a-c. Similarly, the APD's on comparison of the different models with the experimental data are given in Table 5. Of the three systems, the Pinonic acid: $\left(\mathrm{NH}_{4}\right)_{2} \mathrm{SO}_{4}$ saw the greatest reduction in surface tension. There were 5 model combinations employed here. These consisted of an additive approach using both the Tamura mixing rule and Suarez thermodynamic method using the range of values for the surface tension, bulk and critical molar volume. The remaining 3 schemes include an additive approach using the binary $L i L u$ model and a coupled inorganic/organic approach using the "full" LiLu model assuming competing adsorption and no competing adsorption. A coupled inorganic/organic approach simply refers to the treatment of the effect of the inorganic and organic solutes on surface tension within the same theoretical framework. For the calculations of activity coefficients the total water content was used rather than partition to an organic and inorganic fraction. For the Pinonic acid: $\left(\mathrm{NH}_{4}\right)_{2} \mathrm{SO}_{4}$ system the additive scheme using both the Tamura and Suarez methods under-predict the surface tension, the Suarez method producing the largest deviation. The smallest APD is found using the "full" LiLu model which gives an average value of $-4.04 \%$, the deviation increasing with increasing solute mass fraction. Thus it is likely that neglect of the mutual influence both in calculations of nonideality and within the actual model framework create these deviations. For the Succinic acid: $\left(\mathrm{NH}_{4}\right)_{2} \mathrm{SO}_{4}$ system, the reduction in surface tension is not as great. Similarly, the ability of the modelling approaches to capture this slight variability is somewhat better. However, the variation in the Tamura and Suarez model predictions are rather large on comparison with experimental data due to the variations in the pure component surface tension used. Despite good agreement between most model combinations as indicated in Table 5, the smallest APD of $1.85 \%$ is found for an additive approach using the Suarez method. However, the additive approach using the $L i L u$ method (fit to binary data) has a similar APD of $2.36 \%$. The final system of Oxalic acid:Nacl experiences the smallest reduction in surface tension, though for rather small concentrations. In this instance, again there is some variation 

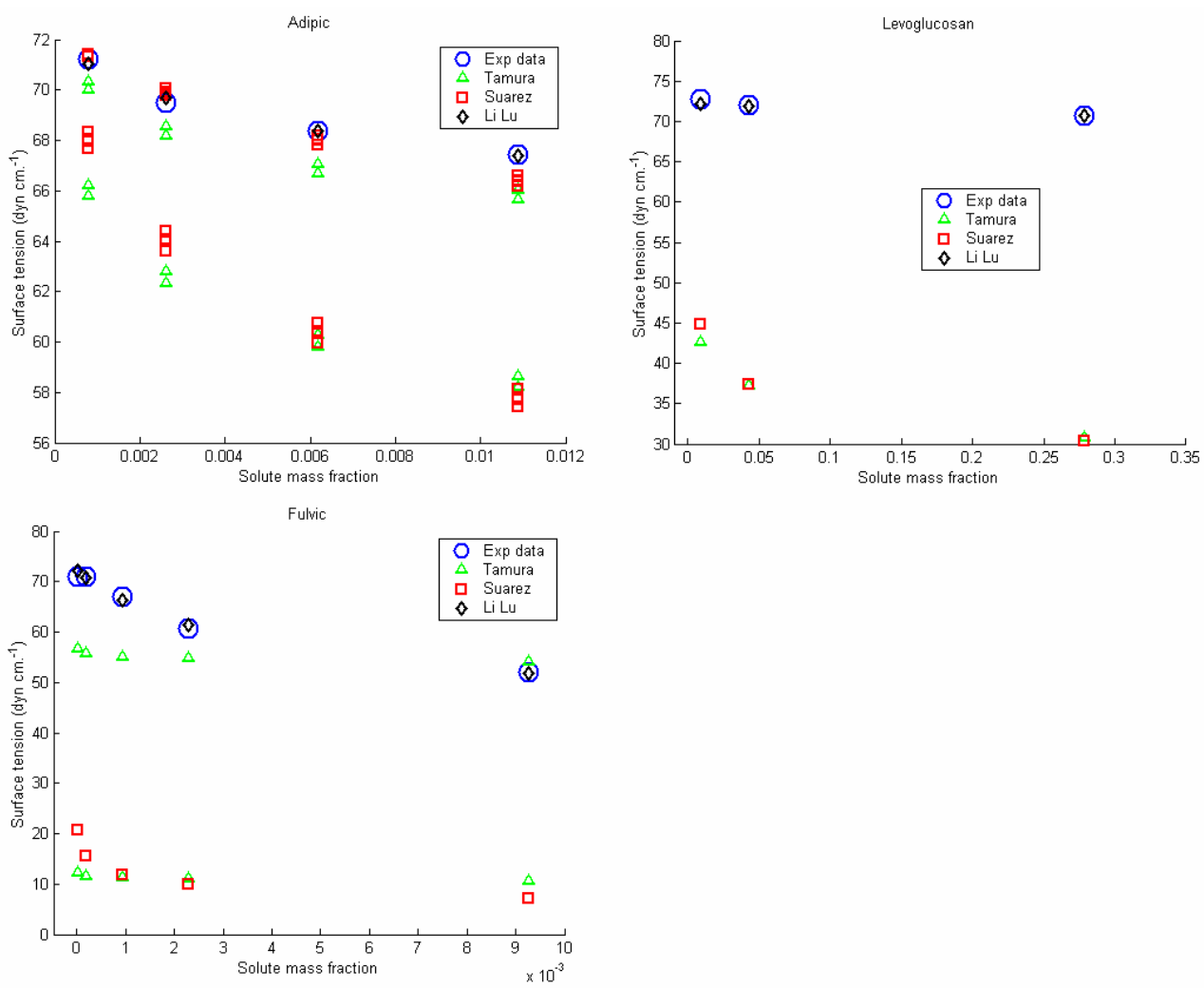

Fig. 1c. Measured surface tension for Adipic, Levoglucosan and Suwanee River Fulvic acid ("Exp data"), versus predictions from the Tamura mixing rule "Tamura" and Suarez thermodynamic method "Suarez". Also shown are predictions from the LiLu thermodynamic model fit to the binary data (" $L i L u$ "). All models are described in the text. Two sets of data represented by the same markers result from essentially two choices of pure component surface tensions within the predictive models.

Table 4. Model combinations applicable to mixed organic and mixed inorganic/organic systems. The column "coupled inorganic/organic method" highlights the method used for coupling the effect of the inorganic and organic fraction on the solution surface tension. The column "Mixed Organic" highlights approaches used to model the surface tension contribution from the organic fraction. The column "Nonideality" highlights whether one assumes activity coefficients in both the inorganic and organic fraction to be the same as one would expect in a binary mixture (semi-ideal) or by a full treatment of solute - solvent interactions (Nonideal). " $L i L u$ version" highlights the form of the $L i L u$ model used for modelling aqueous multicomponent organic mixtures. "Binary organic representation" highlights the binary method sued within the Fainerman-Mille mixing rule.

\begin{tabular}{|c|c|c|c|c|}
\hline Coupled & Mixed Organic & Nonideality & LiLu version & Binary Organic \\
\hline Inorganic/Organic & & & (where applicable) & representation \\
\hline \multirow[t]{2}{*}{ method } & & & & technique \\
\hline & & & & (where applicable) \\
\hline \multirow[t]{3}{*}{ Additive } & $\mathrm{FM}$ & Semi-ideal & NA & Tamura \\
\hline & & Semi-ideal & N:A & Suarez \\
\hline & & Semi-ideal & NA & LiLu \\
\hline \multirow[t]{4}{*}{ Additive } & LiLu & Semi-ideal & No competition & NA \\
\hline & & Semi-ideal & Competition & NA \\
\hline & & Nonideal & No competition & NA \\
\hline & & Nonideal & Competition & NA \\
\hline \multirow[t]{4}{*}{ Coupled LiLu } & $N / A$ & Semi-ideal & No competition & NA \\
\hline & & Semi-ideal & Competition & NA \\
\hline & & Nonideal & No competition & NA \\
\hline & & Nonideal & Competition & NA \\
\hline
\end{tabular}


Table 5. Average percentage deviations on comparison with experimental data for various model combinations applicable to ternary systems (1 organic, 1 inorganic).

\begin{tabular}{|c|c|c|c|c|c|c|c|}
\hline \multirow[t]{2}{*}{ System } & \multirow[t]{2}{*}{ Method } & \multicolumn{3}{|c|}{ Critical molar volume } & \multirow[b]{2}{*}{$V_{c 2}$} & & \multirow[b]{4}{*}{ Molar volume } \\
\hline & & $V_{c 1}$ & & & & & \\
\hline & & & & & & & \\
\hline & & $\sigma_{\text {pure }}$ & & Molar volume & $\sigma_{\text {pure }}$ & & \\
\hline & & $\sigma_{1}$ & $\sigma_{2}$ & & $\sigma_{1}$ & $\sigma_{2}$ & \\
\hline \multirow[t]{5}{*}{ Tern1 } & Tamura & -20.6607 & -14.9368 & $V_{1}$ & --- & --- & \\
\hline & Suarez & -31.5879 & -25.3200 & $V_{2}$ & -- & -- & \\
\hline & LiLu & -4.8056 & & & & & \\
\hline & LiLu (1) & -4.0861 & & & & & \\
\hline & LiLu (2) & -4.0386 & & & & & \\
\hline \multirow[t]{7}{*}{ Tern 2} & |Tamura & -3.2401 & 2.5552 & $V_{1}$ & -3.2401 & 2.5552 & $V_{1}$ \\
\hline & & -3.1369 & 2.5937 & $\sqrt{2}$ & -3.1369 & 2.5937 & $V_{2}$ \\
\hline & Suarez & -6.4490 & 1.8719 & $V_{1}$ & -6.5522 & 1.8615 & $V_{1}$ \\
\hline & & -6.6086 & 1.8561 & $V_{2}$ & -6.7135 & 1.8457 & $V_{2}$ \\
\hline & Li Lu & 2.3585 & & & & & \\
\hline & LiLu (1) & 2.7026 & & & & & \\
\hline & LiLu (2) & 3.1181 & & & & & \\
\hline \multirow[t]{7}{*}{ Tern 3} & Tamura & 0.0174 & 0.6785 & $V$ & 0.0174 & 0.6785 & $V /$ \\
\hline & & 0.0202 & 0.6789 & $V_{2}$ & 0.0202 & 0.6789 & $V_{2}$ \\
\hline & Suarez & -5.2722 & 0.2002 & $V /$ & -5.3811 & 0.1935 & $V /$ \\
\hline & & -5.2959 & 0.1987 & $V_{2}$ & -5.4050 & 0.1921 & $V_{2}$ \\
\hline & Li Lu & 0.5800 & & & & & \\
\hline & LiLu (1) & 1.1991 & & & & & \\
\hline & LiLu (2) & 1.2003 & & & & & \\
\hline \multicolumn{3}{|c|}{ LiLu(1) - No competing adsorption } & & & & & \\
\hline \multicolumn{3}{|c|}{ LiLu(2) - Competing adsorption } & & & & & \\
\hline
\end{tabular}

in the Tamura and Suarez additive approaches, though the additive Tamura approach gives the smallest APD of $0.0202 \%$ for one specific pure component surface tension. In this system, the LiLu model predicts a surface tension very close to that of pure water. It appears that one could model the surface tension of such ternary systems relatively well if one, by chance, uses the correct value of the pure component surface tension for the Tamura and Suarez models in an additive approach. Whilst the graphs indicate qualitatively some discrepancies between the $L i L u$ approach, the absolute deviations are quite small, ranging from 1.2 to $-4.09 \%$.

\subsection{Mixed organic system}

A mixed system consisting of only organic compounds was studied separately ("Multi 1"). The composition is given in Table 1b. For the mixed organic case there are a number of model combinations applicable to the different multicomponent methods. These are listed in Table 6 along with the APD on comparison with experimental data. For simplicity, when using the binary predictive models of Tamura and Suarez, the parameters which resulted in the smallest APD for the appropriate binary systems were employed. As shown in Table 6 and Fig. 3 certain variations of the modelling frameworks can capture the variability very well. Specifically, use of the $F M$ mixing rule with the LiLu binary method and variations of the "full" LiLu model framework have absolute APD's of only 2.27 to $2.26 \%$. This indicates that essentially use of the binary data in the $F M$ mixing rule provides an excellent tool for recapturing the surface tension of this system. Similarly, variations of the $L i L u$ model are just as accurate. However, use of the Tamura and Suarez methods within the $F M$ rule do not fare as well for this system. This is specifically down to the inability of both methods to capture the surface tension effect of fulvic acid. As discussed in Sect. 3.2.2, there is no solution to the Suarez method when using the pure component surface tension of 102.9 dyne $\mathrm{cm}^{-1}$. 
Table 6. Average percentage deviations on comparison with experimental data for various model combinations applicable to a mixed organic system. FM - Fainermann and Miller mixing rule described in the text. LiLu - The Li and Lu thermodynamic scheme described in the text. The column "Nonideality" highlights whether one assumes activity coefficients in both the inorganic and organic fraction to be the same as one would expect in a binary mixture (semi-ideal) or by a full treatment of solute - solvent interactions (Nonideal). "LiLu version" highlights the form of the $\mathrm{LiLu}$ model used for modelling aqueous multicomponent organic mixtures. "Binary organic" highlights the binary method sued within the Fainerman-Mille mixing rule.

\begin{tabular}{|l|l|l|l|r|}
\hline Mixed Organic & Nonideality & LiLu version & Binary Organic & 'Multi 1' \\
\hline FM & Semi-ideal & - & Tamura & -17.923634 \\
\hline & Semi-ideal & - & Suarez & -84.816371 \\
\hline & Semi-ideal & Binary & LiLu & -2.262770 \\
\hline LiLu & Semi-ideal & No competition & N/A & -2.262770 \\
\hline & Semi-ideal & Competition & N/A & -2.262768 \\
\hline & Nonideal & No competition & N/A & -2.262770 \\
\hline & Nonideal & Competition & N/A & -2.262768 \\
\hline
\end{tabular}

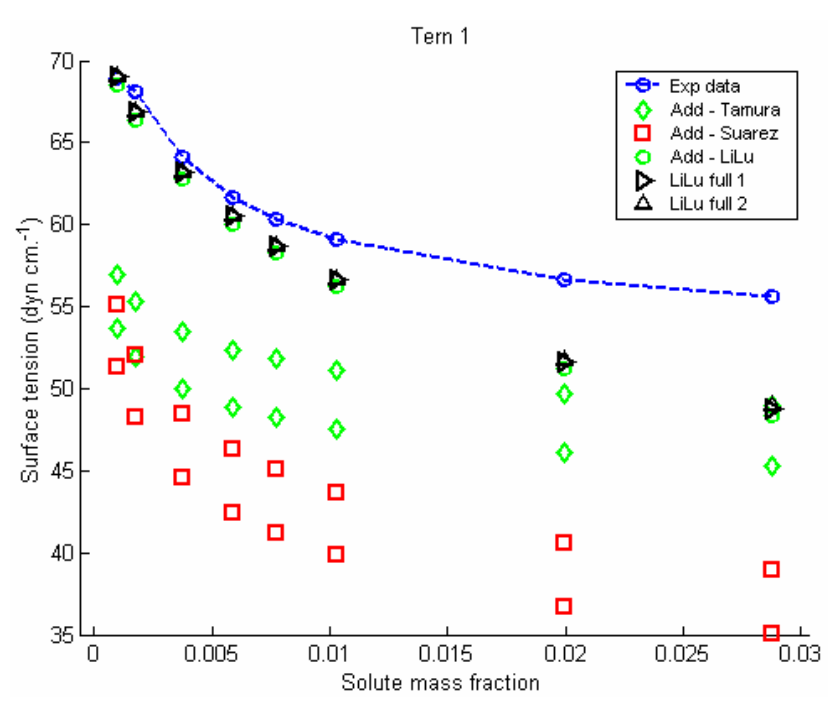

Fig. 2a. Measured surface tension for Pinonic acid/ $\left(\mathrm{NH}_{4}\right)_{2} \mathrm{SO}_{4}$ ("Exp data"), versus predictions from various model combinations. "Add" - adding the deviations from the surface tension of pure water from both the inorganic and organic fraction. "Tamura", "Suarez" and "LiLu" - use of the Tamura mixing rule, Suarez thermodynamic model and the $L i L u$ binary model fit to the binary data to represent the binary organic surface tension. " $L i L u$ full 1 " - Coupled inorganic/organic model assuming no competing adsorption. " $L i L u$ full 2" - Coupled inorganic/organic model assuming competing adsorption.

Results are perhaps even more encouraging when one considers that for comparisons with the experimental data an assumed mass for Suwannee River FA has to be assigned which was based on a suggestive molecular structure. This structure, which has been published previously (Topping et al., 2005b), is based on HNMR analysis. Similarly, in order to calculate the average molar area of solution using Eq. (22), then the simple expression given by Eq. (9) was used, which in itself might be considered to introduce errors. However,

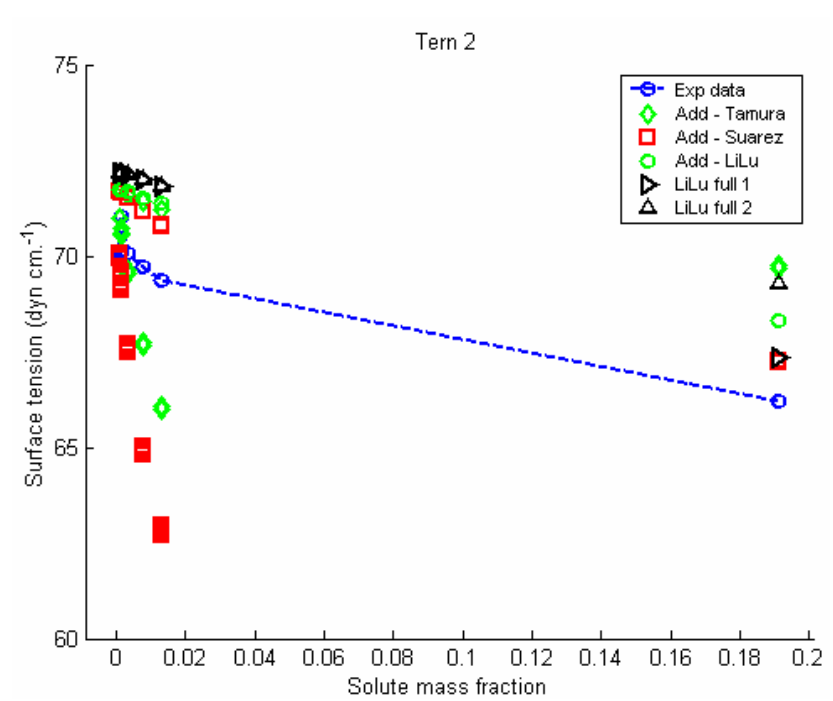

Fig. 2b. Measured surface tension for Succinic acid/ $\left(\mathrm{NH}_{4}\right)_{2} \mathrm{SO}_{4}$ ("Exp data"), versus predictions from various model combinations. "Add" - adding the deviations from the surface tension of pure water from both the inorganic and organic fraction. "Tamura", "Suarez" and " $L i L u$ " - use of the Tamura mixing rule, Suarez thermodynamic model and the LiLu binary model fit to the binary data to represent the binary organic surface tension. "LiLu full 1" - Coupled inorganic/organic model assuming no competing adsorption. "LiLu full 2" - Coupled inorganic/organic model assuming competing adsorption.

Fainerman et al. (2002) have already noted the inherent insensitivity to choice of $\omega$. This is not probed further here.

\subsection{Multi-component inorganic/organic systems}

Three mixed inorganic/organic systems were studied as presented in Table $1 \mathrm{~b}$ and the surface tension behaviour displayed in the Figs. 4a-c. For mixed inorganic/organic systems the models presented here allow 11 different ap- 


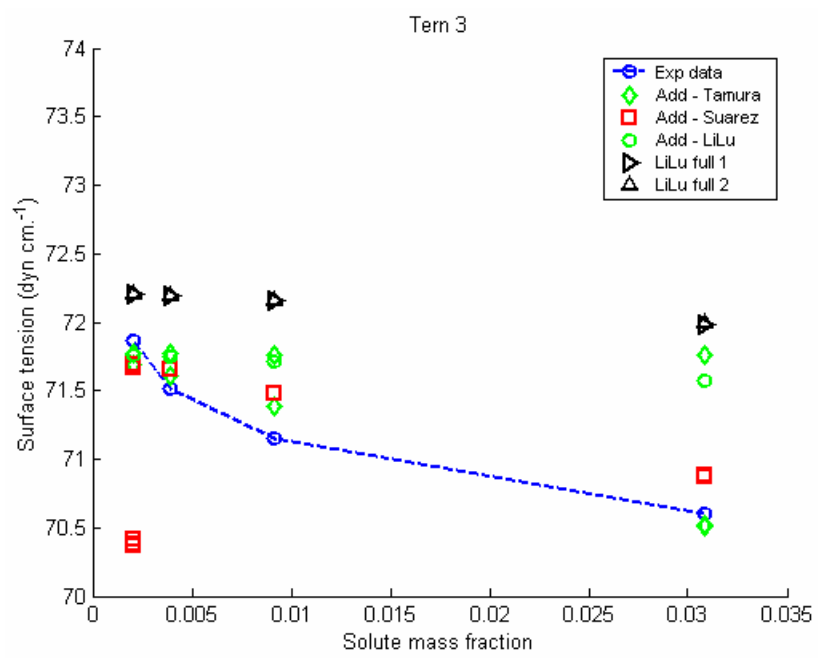

Fig. 2c. Measured surface tension for Oxalic acid/ $\mathrm{NaCl}$ ("Exp data"), versus predictions from various model combinations. "Add" - adding the deviations from the surface tension of pure water from both the inorganic and organic fraction. "Tamura", "Suarez" and " $L i L u$ " - use of the Tamura mixing rule, Suarez thermodynamic model and the LiLu binary model fit to the binary data to represent the binary organic surface tension. "LiLu full 1" - Coupled inorganic/organic model assuming no competing adsorption. " $L i L u$ full 2" - Coupled inorganic/organic model assuming competing adsorption.

proaches which can be broadly categorised into 2 groups: 1) - additive contributions from the separate inorganic and organic fractions and 2) - Use of the LiLu model framework with all the relevant combinations regarding ideality and competition for adsorption. The additive contributions include the use of the $F M$ mixing rule for the organic fraction using the three separate binary models, and the LiLu model applied to both the inorganic and organic fraction separately. All of the combinations are shown in Table 7 along with the associated APD on comparison with experimental data.

For all of the systems studied, the largest deviation from experimental data was found when using an additive approach and the Suarez binary technique in the FM mixing rule. This is ascribed to the large discrepancies of the Suarez technique for modelling the surface tension reduction of fulvic acid as discussed in Sect. 3.2.2. The second largest deviation occurs using the remaining predictive technique of Tamura. However, deviations are much smaller than when using the Suarez method due to reasons discussed above. When using these models, the choice of pure component surface tension and surface area parameters were chosen based on the smallest APD of the binary comparisons as analysed in Sect. 3.2.2. The use of the binary data, represented by the $L i L u$ binary model, within the $F M$ mixing rule and in the full $L i L u$ model produce much smaller APDs. However there are some interesting features from the various model predictions. For the "Multi 2" case, whilst APDs remain low for most

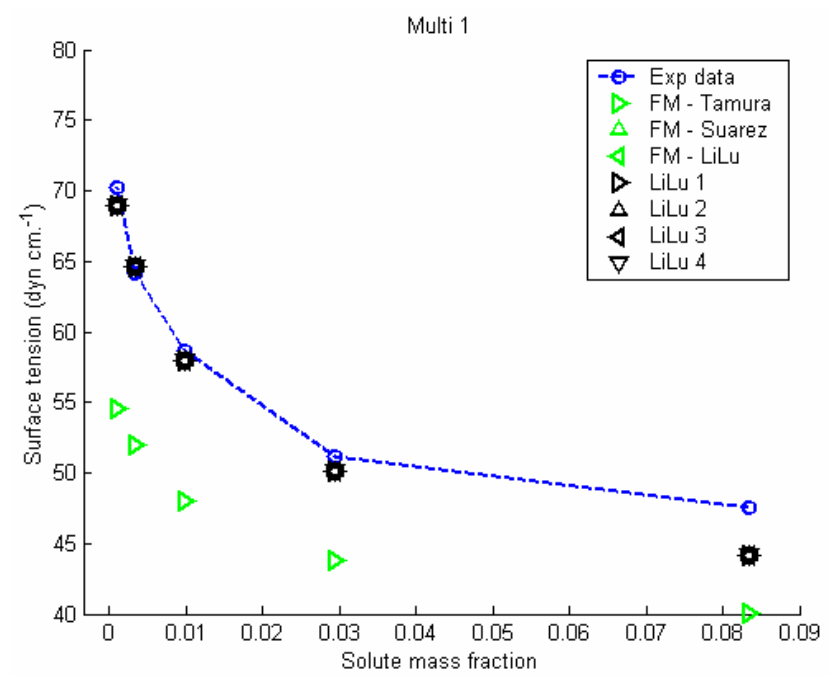

Fig. 3. Measured surface tension for a mixed organic system ("Exp data"), versus predictions from various model combinations. " $F M$ " - multicomponent surface tensions calculated using the Fainermann and Miller mixing rule (see txt).. "Tamura", "Suarez" and " $L i L u$ " - use of the Tamura mixing rule, Suarez thermodynamic model and the $L i L u$ binary model fit to the binary data to represent the binary organic surface tension. "LiLu 1" - LiLu model assuming semiideality and no competing adsorption. " $L i L u 2$ " - LiLu model assuming semi-ideality and competing adsorption. " $L i L u$ 3" - LiLu model assuming nonideality and no competing adsorption. " $\mathrm{LiLu}$ 4" - LiLu model assuming nonideality and competing adsorption.

remaining combinations, at relatively higher concentrations the model which aims to account for competing adsorption and solution non-ideality in the separate fractions, predicts that the surface tension should rise. Also the difference between the additive approach and fully coupled LiLu framework is negligible. A similar yet smaller rise in surface tension is predicted when changing the model to that which does not account for competing adsorption. The smallest APD is found when using the additive approach for the inorganic and organic contributions while employing the full $L i L u$ model to analyse the organic fraction assuming semi-ideality and competing adsorption. It is difficult to tease apart reasons for these results, not least due to issues such as multi-component solute activities for compounds such as fulvic acid using the representative structure within UNIFAC. Similarly, it is beyond the scope of the paper to analyse whether the theoretical grounding of the $L i L u$ model is suitable for such a mixed surfactant system. It is however interesting and encouraging to see that the additive approach, utilising the $F M$ mixing rule and the binary data represented by the LiLu model, works very well in reproducing the experimental data as indicated by the APD of around $-1.02 \%$.

A similar pattern is found for the "Multi 3" case where all model variations, except those using the purely predictive techniques, work very well. In this system there is no 

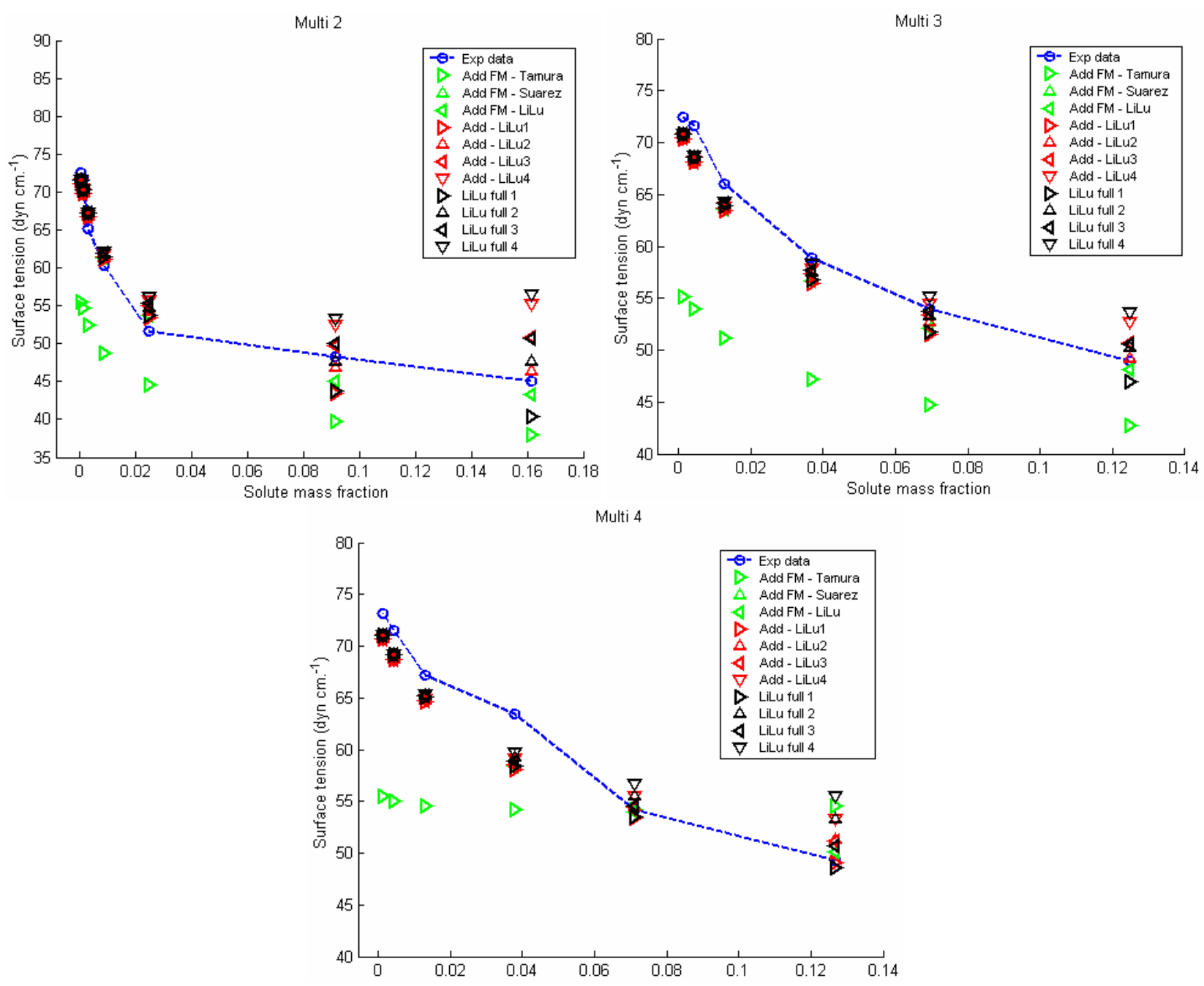

Fig. 4. Measured surface tension for a mixed inorganic/organic system ("Exp data"/relative composition given in Table 1.2), versus predictions from various model combinations. "Add" - adding the deviations from the surface tension of pure water from both the inorganic and organic fraction. "FM" - multi-component organic surface tensions calculated using the Fainermann and Miller mixing rule (see text). "Tamura", "Suarez" and "LiLu" - use of the Tamura mixing rule, Suarez thermodynamic model and the LiLu binary model fit to the binary data to represent the binary organic surface tension. "Add - LiLu" - additive approach using the $L i L u$ model to describe the organic fraction. " $L i L u 1$ " - LiLu model assuming semi-ideality and no competing adsorption. " $L i L u 2$ " - LiLu model assuming semi-ideality and competing adsorption. " $L i L u$ 3" - LiLu model assuming nonideality and no competing adsorption. "LiLu 4" - LiLu model assuming nonideality and competing adsorption. " $L i L u$ full" - Coupled inorganic/organic $L i L u$ model with the same combinations described above

predicted increase in surface tension within the experimental range of concentrations. Using the additive approach, treating the organic fraction within the $L i L u$ model framework, one obtains a range of APDs from -0.73 to $-4.08 \%$. Use of the binary data within the $F M$ mixing rule also results in a small APD of $-3.39 \%$. Again a similar pattern is found for the "Multi 4" case, however in this instance the additive approach employing the Tamura mixing rule produces a relatively constant surface tension. The remaining model variations on the other hand capture the variability well. The additive approach employing the $L i L u$ model to describe the organic fraction produces APDs ranging from -1.16 to $-3.59 \%$, whereas the coupled $L i L u$ model results in APDs ranging from 0.40 to $-3.32 \%$.

Thus it would appear that use of binary data is crucial to capturing the behaviour of multi-component surface tensions for the systems studied here. Whilst the applicability of the coupled thermodynamic model and its various combinations seems to be composition dependent, and produce some interesting if un-validated features with increasing concentration, the direct use of binary data within the $F M$ mixing rule also reproduces measured behaviour very well. This would be 
Table 7. Average percentage deviations on comparison with experimental data for various model combinations applicable to a mixed inorganic/organic system. FM - Fainermann and Miller mixing rule described in the text. LiLu - The Li and Lu thermodynamic scheme described in the text. Coupled $L i L u$ - a complete $L i L u$ model framework which considers both inorganic and organic components. The column "coupled inorganic/organic method" highlights the method used for coupling the effect of the inorganic and organic fraction on the solution surface tension. The column "Mixed Organic" highlights approaches used to model the surface tension contribution from the organic fraction. The column 'Nonideality' highlights whether one assumes activity coefficients in both the inorganic and organic fraction to be the same as one would expect in a binary mixture (semi-ideal) or by a full treatment of solute - solvent interactions (Nonideal). "LiLu version" highlights the form of the LiLu model used for modelling aqueous multicomponent organic mixtures. "Binary organic representation" highlights the binary method sued within the Fainerman-Mille mixing rule.

\begin{tabular}{|c|c|c|c|c|c|c|c|}
\hline Coupled & Mixed Organic & Nonideality & LiLu version & Binary Organic & & & \\
\hline Inorganic/Organic & & & (where applicable) & representation & & & \\
\hline \multirow[t]{3}{*}{ method } & & & & technique & & & \\
\hline & & & & (where applicable) & & & \\
\hline & & & & & 'Multi 2' & 'Multi 3' & 'Multi 4' \\
\hline \multirow[t]{3}{*}{ Additive } & FM & Semi-ideal & NA & Tamura & -18.997137 & -20.138267 & -11.607360 \\
\hline & & Semi-ideal & NA & Suarez & -83.746370 & -84.209630 & -83.523691 \\
\hline & & Semi-ideal & NVA & LiLu & -1.018316 & -3.395110 & -2.940407 \\
\hline \multirow[t]{4}{*}{ Additive } & LiLu & Semi-ideal & No competition & NMA & -2.580254 & -4.080060 & -3.593119 \\
\hline & & Semi-ideal & Competition & NA & 0.543249 & -2.770457 & -2.333630 \\
\hline & & Nonideal & No competition & NAA & 3.096647 & -1.913680 & -2.368826 \\
\hline & & Nonideal & Competition & NAA & 5.606147 & -0.712719 & -1.164764 \\
\hline \multirow[t]{4}{*}{ Coupled LiLu } & N/A & Semi-ideal & No competition & NA & -1.986098 & -3.594710 & -3.318168 \\
\hline & & Semi-ideal & Competition & NAA & 1.716793 & -1.773760 & -0.855337 \\
\hline & & Nonideal & No competition & NAA & 3.690807 & -1.428344 & -2.093820 \\
\hline & & Nonideal & Competition & NYA & 6.746243 & 0.260997 & 0.402119 \\
\hline
\end{tabular}

the model of choice when binary data is available. In the following section, the sensitivity to predictions of the critical saturation ratio are analysed.

\section{Critical supersaturations}

In the following analysis, activation predictions are calculated using ADDEM (Topping et al., 2005a, b) based on the surface tension modelling techniques described in the previous sections whilst neglecting bulk to surface partitioning (Sorjamaa et al., 2004). Specifically this will help to elucidate on the importance of capturing composition dependent surface tensions in a fundamental Kohler theory approach. In Sect. 5.1 two case studies are used to analyse the possible effect of bulk to surface partitioning on both the solute and Kelvin effect within Kohler theory.

Here the model ADDEM is taken into the supersaturated humid regime by applying model adjustments and extensions which allow growth factor calculations, as well as activation predictions, above $100 \%$ RH. This framework describes the equilibrium of water vapour alone. The details of ADDEM and its use in the sub-saturated humid regime are given in detail in two papers (Topping et al., 2005a, b). For treating non-ideality, both the Pitzer-Simonson-Clegg (Clegg and Pitzer, 1992) and UNIFAC model (Fredenslund et al., 1975) are employed in an additive approach for treating mixed inorganic/organic systems. The advantages and caveats of such an approach have been discussed previously and the complexities in treating mixed inorganic/organic systems are treated extensively in the literature and will not be reviewed here (e.g. see Clegg and Seinfeld, 2006; Clegg et al., 2001, and references therein). For this study, the updated interaction parameters of Peng et al. (2001) were used in combination with the original matrix of Hansen et al. (1991) to retain consistency with the surface tension calculations. Whilst some theoretical studies suggest the effect of highly surface active compounds may alter the water activity by decreasing the effective number of molecules in the Raoult term, experimental studies for atmospherically relevant species have not been carried out nor has the effect of non-ideality been explored, and as such is not considered here (Sorjamaa et al., 2004).

When developing the mathematical framework required here, it is at first necessary to define what information one requires to extract from the Köhler curve; for example, an equilibrium radius for a given super-saturation ratio or the critical point on the Köhler curve. For the latter case, the required one dimensional search uses the control of water activity in Eq. (1) to define firstly upper and lower boundaries which bracket the critical point. Once the model is run with a given water activity $\left(a_{w}\right)$ then the appropriate physical information such as surface tension and density can be calculated and the point on the Köhler curve determined. Unfortunately derivative information cannot be attained easily which would be required to define the point at which the derivative of the Köhler curve with respect to radius becomes zero (the critical point). Specifically, the use of complex thermodynamic activity coefficient models and surface tension rules would require complicated derivative information. Fortunately there is no need to derive such relationships and one can use methods that need only evaluations of the function such as a basic bisection approach or Brent's method for function minimisation. The latter is likely to be particularly useful since it 
will exploit the parabolic nature of the Köhler curve near the critical point.

Applying the above technique, Figs. 5a-8a show the critical saturation ratio as a function of dry size for all of the mixed cases studied in Sect. 4.3 and Sect. 4.4, whereas Figs. $5 b-8 b$ show the surface tension values at the critical points. The deviation between predicted critical points increases as the size of the dry particle decreases. Despite increased convergence at larger dry sizes, thus more dilute droplets (reduced Kelvin effect), there is still a noticeable difference between assuming the surface tension is that of pure water and explicitly taking into account the influence of solutes.

In all systems the largest and lowest predicted critical points are given when using the surface tension of pure water and employing purely predictive techniques respectively. At 10, 100 and $500 \mathrm{~nm}$ dry diameter the difference in critical saturation between both techniques for "multi 1" was 6.46, 0.139 and $0.0093 \%$ respectively. Larger deviations were found for "multi 2" (8.27, 0.186 and $0.0141 \%)$. However, the remaining systems exhibited smaller deviations (5.22, 0.0109 and $0.0066 \%$ for "multi 3"; $1.78,0.047$ and $0.004 \%$ for "multi 4"). Looking at the deviation between the other modelling techniques one can see that, whilst Table 7 indicates relatively small average percentage deviations on comparison between models and experimental surface tension data, propagating these deviations through to calculations of the critical point leads to significant differences on decreasing dry size. At larger sizes, all model combinations converge, which is to be expected given the surface tension behaviour already described in earlier sections, and evident in Figs. 3-4 whereby all models converge at low solute concentrations.

An interesting feature which is apparent in each system is the sudden drop in the critical point as a function of dry size for certain model combinations. However, Figs. $5 b-8 b$ show that this discrepancy is caused by an inflection in the predictions of surface tension. As discussed earlier, some model combinations did seem to predict that surface tension would increase beyond and toward the end of the range of experimental data. At the smaller dry sizes, where solutions are more concentrated at the critical point, this causes the discrepancy observed. In order to explain this, a more detailed analysis of the surface tension predictions was carried out. On closer inspection, it was found that the multi-component methods were relying on contributions to the mixed surface tension for concentrations beyond which had been used for binary surface tension of fulvic acid. Thus, as shown in Fig. 9, this caused an increase in the mixed surface tension of varying magnitudes. To bypass this problem one requires a smooth function. To this end, following previous studies (Abdul-Razzak and Ghan, 2004), the Szyskowski equation was fit to the binary data for fulvic acid, which is a function of the solute mole fraction:

$$
\sigma_{w s}=\sigma_{w}-0.06473 T(\log 10(1+45586.87346 x))
$$

where $\sigma_{w}$ is the surface tension of pure water and $x$ the solute mole fraction. The range of concentrations used here was between 0.0092 to 0.000019 solute mass fraction. The two binary parameters were found using the same iterative procedure as described earlier. Figure 9 shows the ability of the fitted Szyskowski equation to reproduce the measured data. Use of the new binary representation in the $F M$ mixing rule and the effect on activation predictions is shown in Figs. 5a$8 \mathrm{a}$. One can see that the critical saturation ratio versus dry size is now smooth, all curves converging at larger dry sizes. It is of course questionable whether it is appropriate to model surface tensions beyond the solubility limit of certain compounds. This example clearly illustrates the effect when such limitations are manifest in the data, which in this instance creates a discontinuity in model predictions. These results indicate that the use of theoretical frameworks which contain parameters derived from binary data may predict unphysical behaviour when taken beyond the concentration ranges used to fit such parameters.

Thus results suggest that the composition dependent surface tension is a crucial parameter for calculations of the critical point. Similarly, the sensitivity to different models and variations in composition seem to increase with decreasing particle size. For smaller dry sizes, in this study below $40 \mathrm{~nm}$, one would have to make sure that smooth functions of binary surface tension at appropriate concentration ranges were available for use in multi-component methods. Calculations carried out here would suggest that one cannot assume the surface tension of pure water in a consistent theoretical framework. Of course, comparison with laboratory measurements of activation is required on such systems before completely robust conclusions can be made. Ideally this should also include further separate investigations into the solute effect encompassed within the Raoult term. However, the sensitivity when using accurate surface tension models in fundamental Köhler theory is clear.

\subsection{Bulk to surface partitioning}

Calculating the equilibrium composition using all variations of Kohler theory has in the past assumed that the total solute concentrations define both the water activity and surface tension. This was employed in the previous section using the fundamental Kohler equation. Recently however the neglect of bulk to surface phase partitioning has been investigated in various studies (e.g. Sorjamaa and Laaksonen, 2006; Kokkola et al., 2006 and references therein). In the following section the critical saturation ratio of two systems presented in this report are analysed with this effect in mind. To understand this process it is important to understand that an alteration of the surface tension is caused by changing concentration gradients approaching the surface. However, in 
a)

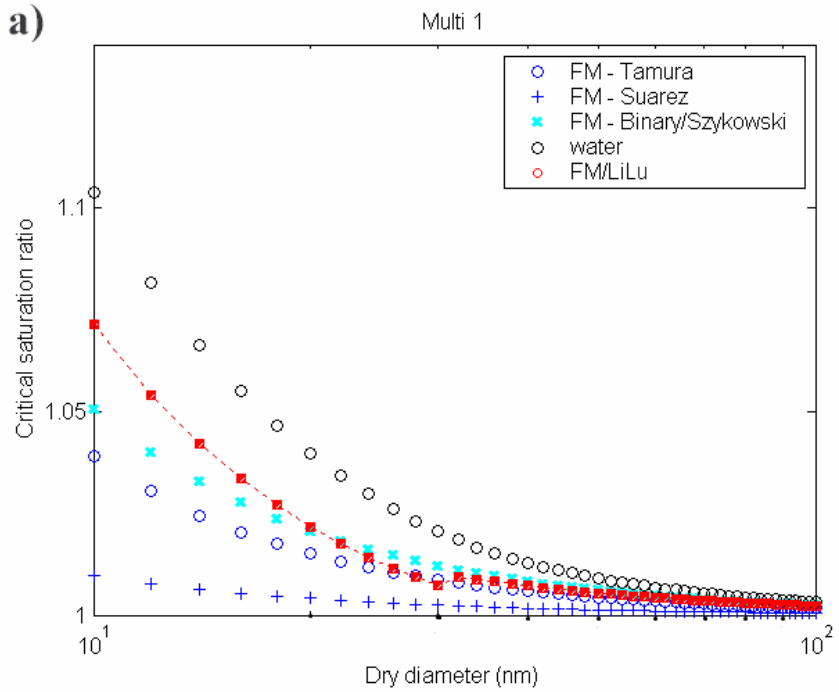

b)

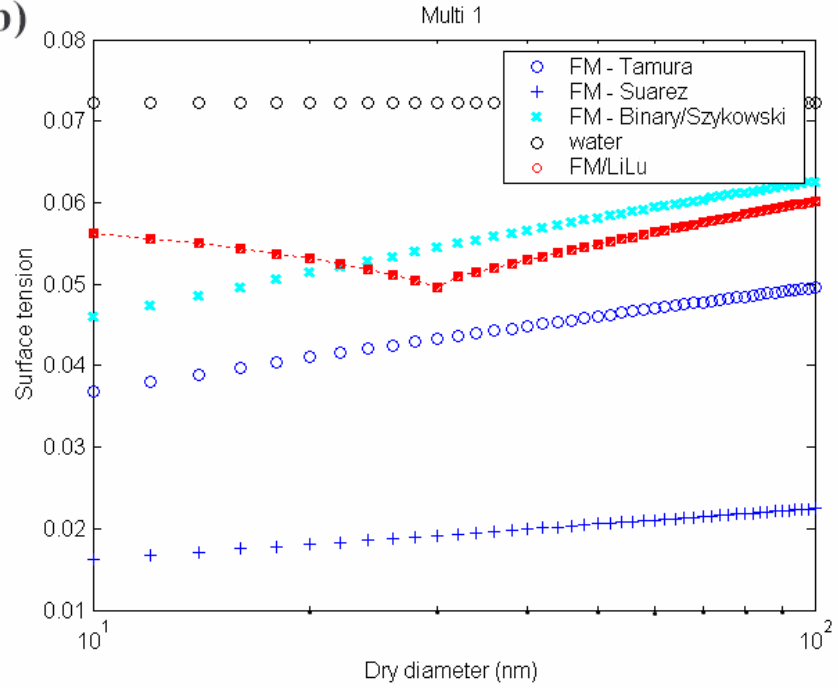

Fig. 5. (a) Critical saturation ratio versus dry diameter for the "multi 1" composition using a variety of surface tension models. (b) Surface tension at the critical point. " $F M$ " - multi-component organic surface tensions calculated using the Fainermann and Miller mixing rule (see text). "Tamura" and "Suarez" - use of the Tamura mixing rule and Suarez thermodynamic model to represent the binary organic surface tension within the Fainermann and Miller mixing rule. "Binary/Szyskowski" - use of the binary LiLu model to represent the binary organic tension of all organic components except Suwannee River Fulvic acid where the Szyskowski equation is employed. "water" - using the surface tension of pure water; " $F M / L i L u$ " - use the $L i L u$ model within the Fainermann Miller mixing rule and the full $L i L u$ model (and all variations thereof). These lines show that the use of a theoretical model fit to binary data may produce unrealistic physical behaviour when taken outside the range of experimental data.

a)

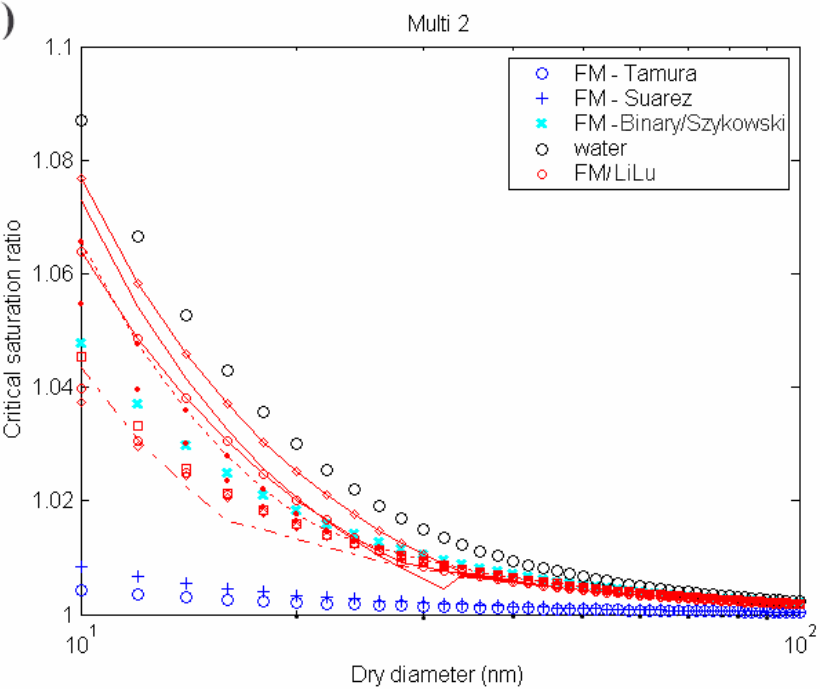

b)

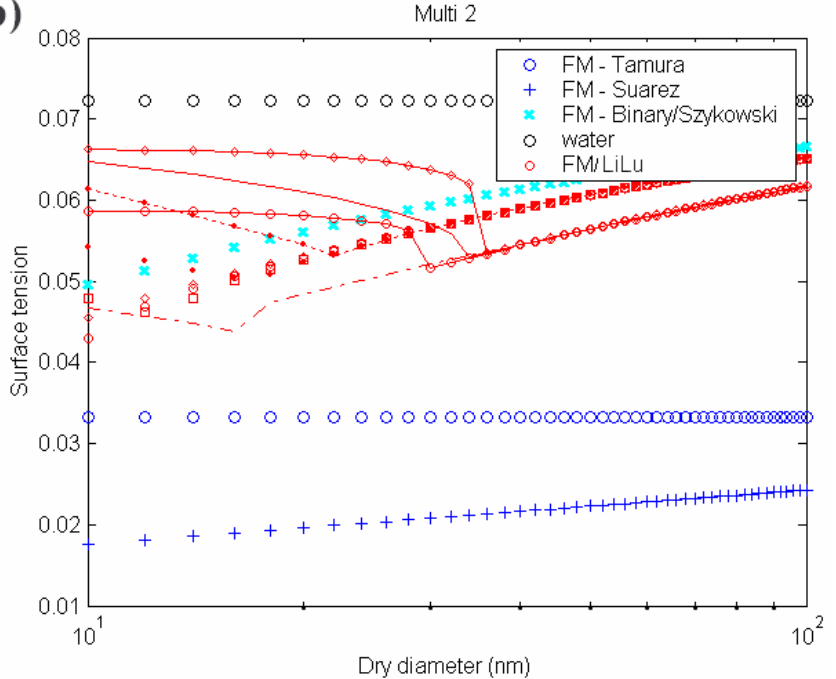

Fig. 6. (a) Critical saturation ratio versus dry diameter for the "multi 2" composition using a variety of surface tension models. (b) Surface tension at the critical point. " $F M$ " - multi-component organic surface tensions calculated using the Fainermann and Miller mixing rule (see text). "Tamura" and "Suarez" - use of the Tamura mixing rule and Suarez thermodynamic model to represent the binary organic surface tension within the Fainermann and Miller mixing rule. "Binary/Szyskowski" - use of the binary LiLu model to represent the binary organic tension of all organic components except Suwannee River Fulvic acid where the Szyskowski equation is employed. "water" - using the surface tension of pure water; "FM/LiLu" - use the $L i L u$ model within the Fainermann Miller mixing rule and the full $L i L u$ model (and all variations thereof). These lines show that the use of a theoretical model fit to binary data may produce unrealistic physical behaviour when taken outside the range of experimental data. 
a)

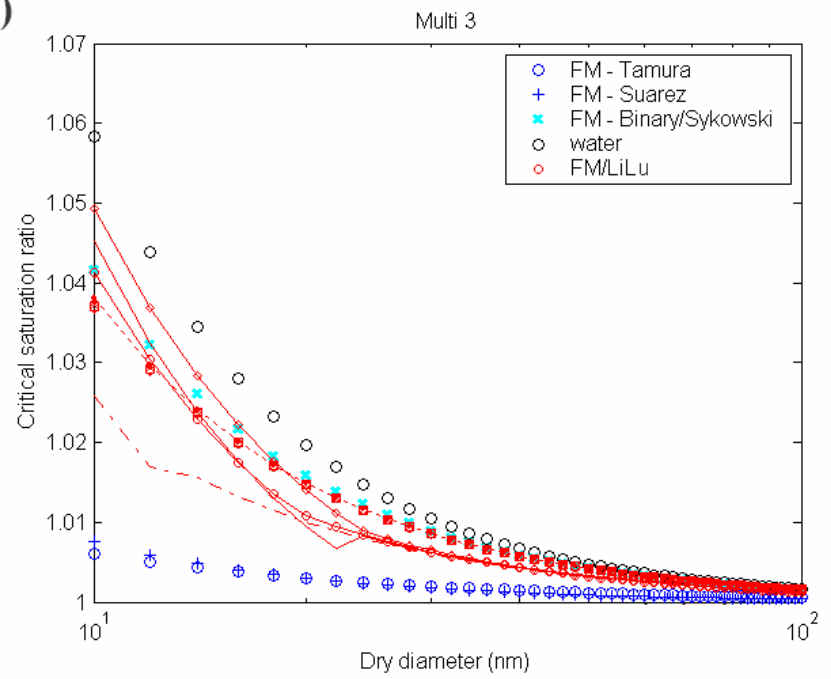

b)

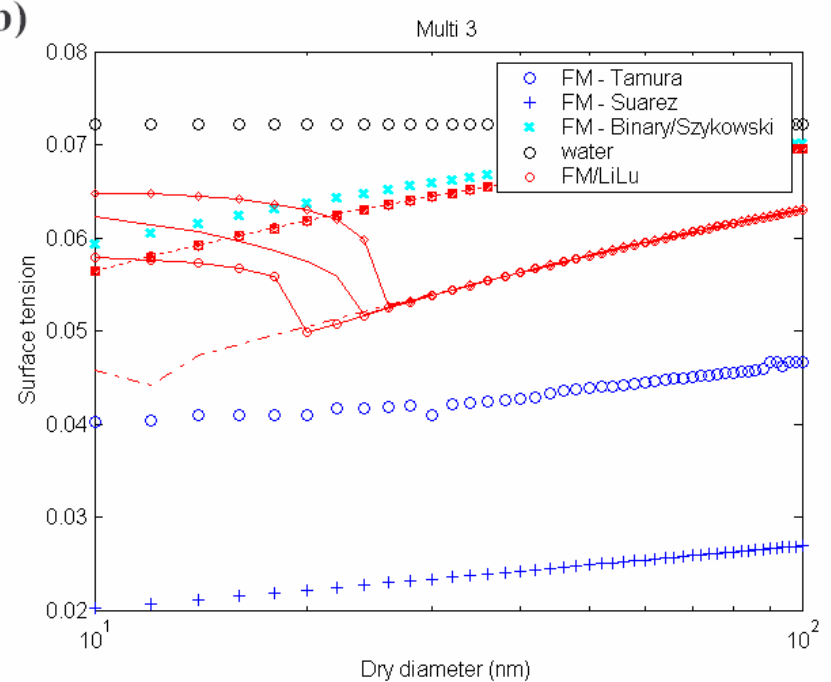

Fig. 7. (a) Critical saturation ratio versus dry diameter for the "multi 3" composition using a variety of surface tension models. (b) Surface tension at the critical point. " $F M$ " - multi-component organic surface tensions calculated using the Fainermann and Miller mixing rule (see text). "Tamura" and "Suarez" - use of the Tamura mixing rule and Suarez thermodynamic model to represent the binary organic surface tension within the Fainermann and Miller mixing rule. "Binary/Szyskowski" - use of the binary LiLu model to represent the binary organic tension of all organic components except Suwannee River Fulvic acid where the Szyskowski equation is employed. "water" - using the surface tension of pure water; " $F M / L i L u$ " - use the $L i L u$ model within the Fainermann Miller mixing rule and the full $L i L u$ model (and all variations thereof). These lines show that the use of a theoretical model fit to binary data may produce unrealistic physical behaviour when taken outside the range of experimental data.

a)

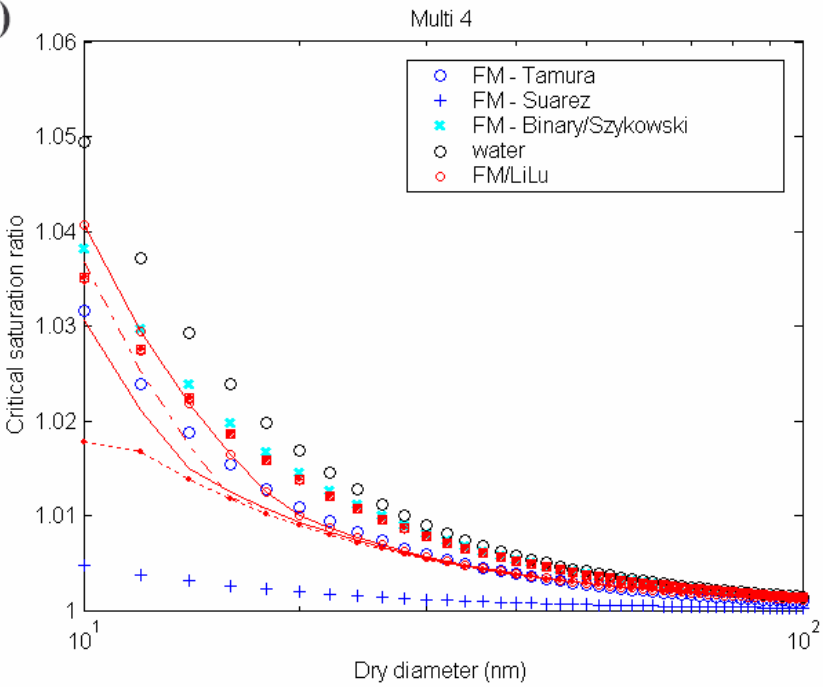

b)

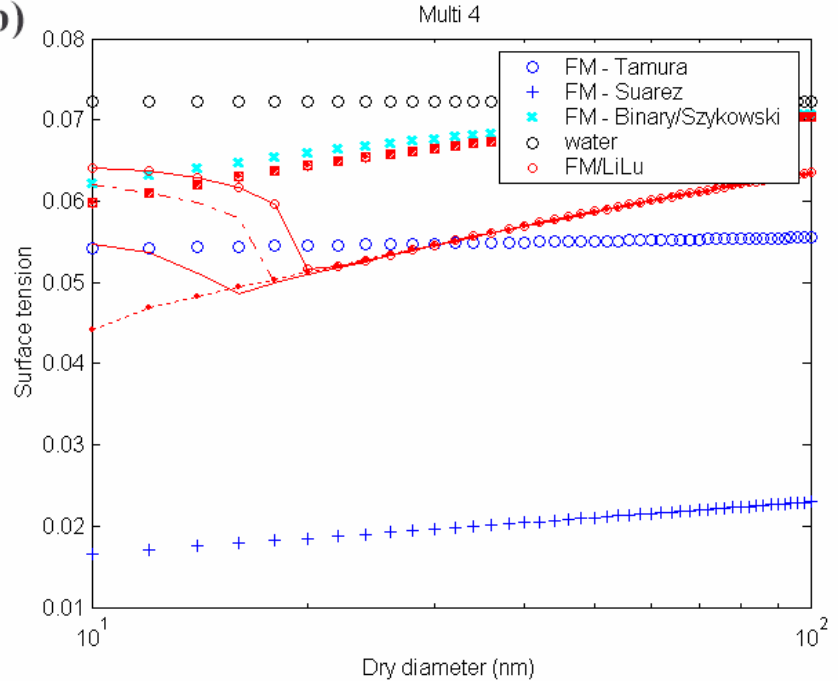

Fig. 8. (a)- Critical saturation ratio versus dry diameter for the "multi 4" composition using a variety of surface tension models. (b) Surface tension at the critical point. "FM" - multi-component organic surface tensions calculated using the Fainermann and Miller mixing rule (see text). "Tamura" and "Suarez" - use of the Tamura mixing rule and Suarez thermodynamic model to represent the binary organic surface tension within the Fainermann and Miller mixing rule. "Binary/Szyskowski" - use of the binary LiLu model to represent the binary organic tension of all organic components except Suwannee River Fulvic acid where the Szyskowski equation is employed. "water" - using the surface tension of pure water; " $F M / L i L u$ " - use the $L i L u$ model within the Fainermann Miller mixing rule and the full $L i L u$ model (and all variations thereof). These lines show that the use of a theoretical model fit to binary data may produce unrealistic physical behaviour when taken outside the range of experimental data. 


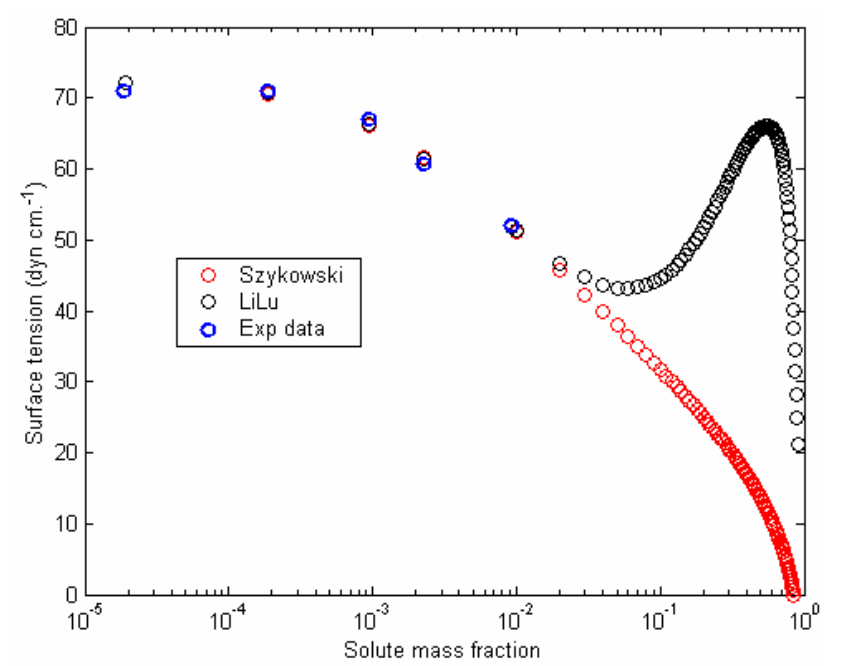

Fig. 9. Measured surface tension for Suwannee River fulvic acid ("exp data") with extended predictions from the $L i L u$ binary model, fit to measured data (" $L i L u$ "), and from the Szyskowski equation fit to the binary data ("Szyskowski”).

Gibbs surface thermodynamics this surface phase between the homogeneous liquid and vapour phase is taken to be infinitely thin allowing an exact definition of the droplet radius (Sorjamaa and Laaksonen, 2006). Any extra material which is related to the change in surface tension is described using surface excess quantities which can be positive or negative (Eq. 32):

$n_{i}^{t}=n_{i}^{b}+n_{i}^{s}$

Where $n_{i}^{t}$ is the total amount of component " $i$ ", $n_{i}^{b}$ and $n_{i}^{s}$ the bulk and surface phase concentrations respectively. The Gibbs adsorption equation relates the change in surface energy to changes in thermodynamic variables and excess quantities (Eq. 33):

$$
\sum_{i} n_{i}^{s} d \mu_{i}+A_{\mathrm{drop}} d \sigma_{w s}=0
$$

Where $\mu_{i}$ is the chemical potential of component " $i$ " , $A_{\mathrm{drop}}$ the surface area of the droplet and $\sigma_{w s}$ the surface tension. The size of the droplet is important since as the surface to volume ratio increases then depletion of material from the bulk becomes important for thermodynamic properties (Sorjamaa and Laaksonen, 2006). Li et al. (1998) studied the effect this can have on the surface tension of small droplets whereas Sorjamaa and Laaksonen (2006) and Kokkola et al. (2006) recently studied the parallel effect on water activity, the latter study focusing on humic like material. The authors devised a numerical technique for binary organic systems in water and ternary mixed inorganic/organic systems in water. Figure 10a shows the effects bulk to surface partitioning calculations for a binary system consisting of Suwannee River fulvic acid in water. Here a solution to the Gibbs adsorption equation (Eq. 33) is found by assuming the surface excess of water is zero following Li et al. (1998). The gradient of surface tension is found by employing the Szyskowski equation already presented in Sect. 5 and activity gradients were calculated using the original UNIFAC framework and the parameters of Hansen et al. (1991). Clearly, using the above considerations results in a significant increase in the predicted critical point. Including the effects of partitioning on calculations of surface tension and water activity leads to an increase in the critical super-saturation of $91.19,37.28$, 15.97 and $3.88 \%$ at $40,100,200$ and $500 \mathrm{~nm}$ dry diameter respectively. The figure also shows predicted critical points if one does not treat bulk to surface partitioning for calculations of both water activity and surface tension, the latter assumed equal to that of pure water. Interestingly, these predictions are closer to the "full" partitioning calculations than those where one accurately captures the variation of surface tension with concentration alone. For example, increases in the critical super-saturation of $13.55,9.88,4.08$ and $0.33 \%$ at 40 , 100, 200 and $500 \mathrm{~nm}$ dry diameter respectively were found when assuming the surface tension of pure water compared to the "full" partitioning calculations. A similar result was found by Sorjamaa and Laaksonen (2006a) for sodium dodecyl sulphate and more recently by Kokkola et al. (2006) for humic like material. For ternary systems Sorjamaa and Laaksonen (2006) introduced the constraint that the ratio of water and salt molecules is practically constant as a function of radius in order to solve the appropriate set of equations. Since we are dealing with a multi-component surfactant mixture in this instance we have not used this approach. Instead we have assumed that the behaviour of each organic behaves as it would in a binary mixture. The consequences of using such an assumption will form the focus of future work, as will the influence of including inorganic compounds in systems more complex than ternary mixtures. As before, a solution to the Gibbs adsorption equation (Eq. 33) is found by assuming the surface excess of water is zero following Li et al. (1998). The gradient of surface tension is found by employing the Szyskowski equation and activity gradients were calculated using the original UNIFAC and the parameters of Hansen et al. (1991). Figure 10b shows again that the effects bulk to surface partitioning calculations clearly results in a significant increase in the predicted critical point. In this instance an increase in the critical super-saturation of $63.98,31.77$, 16.62 and $5.85 \%$ is found for 40, 100, 200 and $500 \mathrm{~nm}$ dry diameter respectively. Interestingly the difference between "full" partitioning calculations and using the total concentrations combined with the surface tension of pure water is very small. As the figure shows both lines are nearly identical with differences of only $2.38,2.54,2.15$ and $1.21 \%$ at 40 , 100,200 and $500 \mathrm{~nm}$ dry diameter respectively.

The above analysis clearly illustrates that a consideration of bulk to surface partitioning can lead to a significant increase in the calculated critical point. As already discussed in previous studied in the literature this will depend on the composition and type of organic being studied. Whilst only 


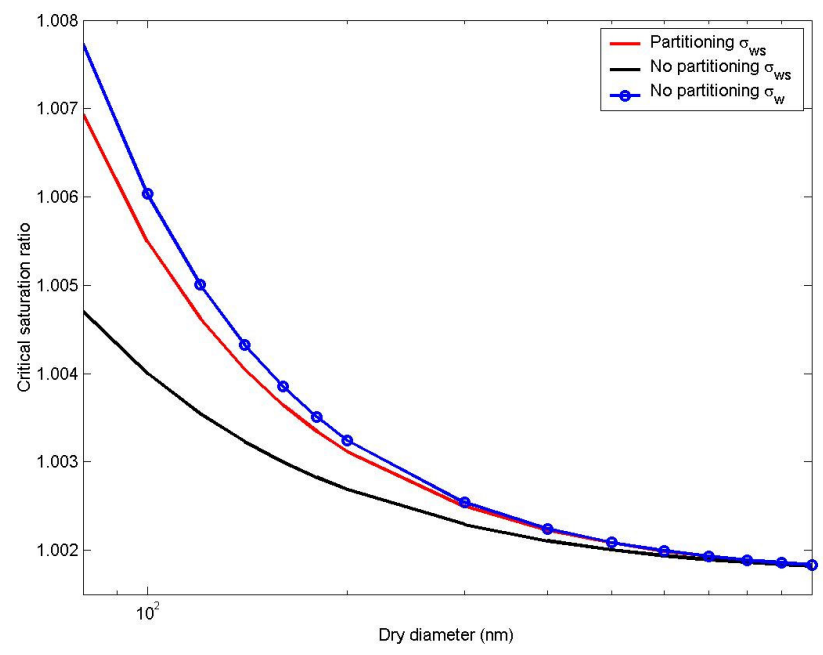

Fig. 10a. Critical saturation ratio versus dry diameter for Suwannee River Fulvic acid. The black line represents use of total solute concentrations for calculating water activity and surface tension which is represented by the Szyskowski equation. The red line represents calculations which take bulk to surface partitioning into consideration for calculation of both water activity and surface tension. The blue line represents calculations based on using total solute concentrations to define the water activity but assuming the surface tension is equal to that of pure water.

two case studies were studied above, a more detailed analysis will form the focus of future work. For example, it is likely that the choice of method for calculating surface tension will be important as in Sect. 5 due to different gradients input into Eq. (33). Despite this however, results presented in this paper still highlight the need for detailed laboratory studies in order to validate model predictions and also to elucidate on processes pertinent to describing how an aerosol particle activates into a cloud droplet.

\section{Summary and conclusions}

The complexity of the organic fraction warrants the analysis of predictive frameworks in order to understand better our ability to model aerosols from various environments. Whilst the qualitative effect of organic compounds on solution surface tensions is understood, our quantitative understanding on mixed organic and mixed inorganic/organic systems is limited. This in itself warrants further laboratory studies which should work in conjunction with ambient measurements of both the chemical and physical properties of aerosol particles. All of the systems studied here showed a reduction in surface tension with increasing solute concentration.

For the ternary mixtures of Oxalic acid: $\mathrm{NaCl}$ and Succinic acid: $\left(\mathrm{NH}_{4}\right)_{2} \mathrm{SO}_{4}$ the reduction in surface tension is quite small. On the other hand, the Pinonic acid: $\left(\mathrm{NH}_{4}\right)_{2} \mathrm{SO}_{4}$ system produced a significant reduction in surface tension. An

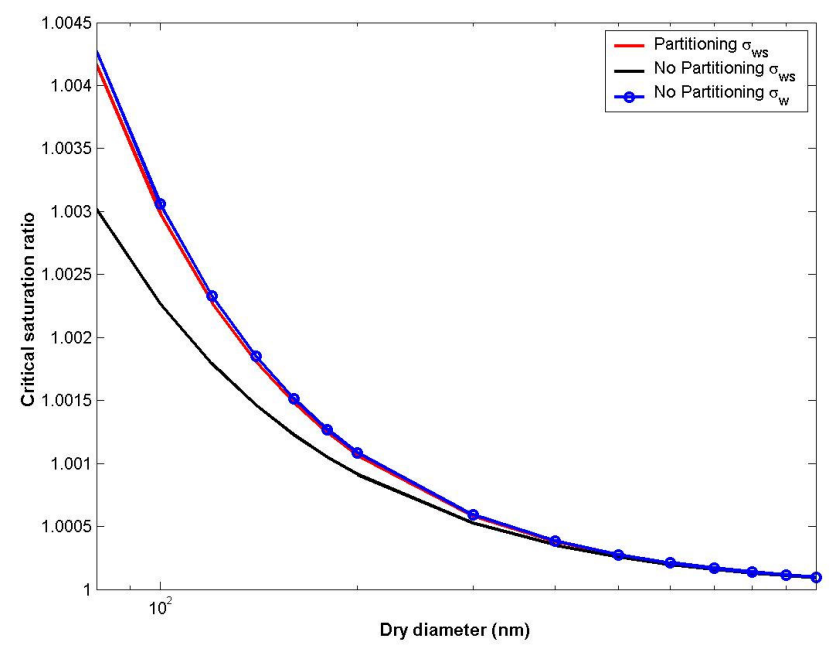

Fig. 10b. Critical saturation ratio versus dry diameter for the "multi 1 " composition. The black line represents use of total solute concentrations for calculating water activity and surface tension which is represented by the Szyskowski equation within the Fainermann Miller mixing rule. The red line represents calculations which take bulk to surface partitioning into consideration for calculation of both water activity and surface tension. The blue line represents calculations based on using total solute concentrations to define the water activity but assuming the surface tension is equal to that of pure water.

analysis of predictions from different model combinations suggested that one could, by chance, capture the variability using a predictive scheme. However this was composition dependent as, for example, the predictive schemes overpredicted the reduction in surface tension for the Pinonic acid: $\left(\mathrm{NH}_{4}\right)_{2} \mathrm{SO}_{4}$ system. Interestingly, for these relatively simple systems then a simple additive approach, using a model fit to the binary data, resulted in average percentage deviations of -4.8 to $0.58 \%$ dependent on the composition.

Analysis of a multi-component organic system found that use of completely predictive technique resulted in large average percentage deviations on comparison with experimental data. However the use of binary data or a coupled thermodynamic model improved predictions significantly. On addition of inorganic solutes the ability of the entirely predictive techniques did not improve. Generally the use of a coupled thermodynamic framework reproduced measured data very well, the best model permutation seeming composition dependent. Similarly, it was found that 'direct' use of binary data within a mixing rule worked excellently as well. Thus it appears that in order to model multi-component surface tensions one requires the use of the appropriate binary data.

The models used in this study were then employed to calculate critical saturation ratios as a function of dry size for all of the multi-component systems studied by first neglecting the possible effect of bulk to surface partitioning. It was clear that deviations between predictions increased as the dry 
size decreased. Similarly, it was evident that using the surface tension of pure water, rather than calculate the influence of the solutes explicitly, lead to a consistently higher value of the critical saturation ratio. Indeed, a neglect of the compositional effects lead to noticeable differences even at large dry sizes. Further analysis clearly illustrates that a consideration of bulk to surface partitioning can lead to a significant increase in the calculated critical point. By employing various assumptions it was possible to perform calculations not only for a binary system but also for a mixed organic system. As already discussed in previous studies in the literature the net effect will depend on the composition and type of organic being studied. A more detailed analysis will form the focus of future work. Despite this however, results presented in this paper still highlight the need for detailed laboratory studies in order to validate model predictions and also to elucidate on processes pertinent to describing how an aerosol particle activates into a cloud droplet.

Acknowledgements. D. Topping is currently funded by DIAC/NCAS and this work was sponsored by the ACCENT Joint Research Programme on Aerosols: Air quality \& Climate. The authors would also like to thank R. Sorjamaa for her useful input regarding bulk to surface partitioning calculations.

Edited by: M. Kulmala

\section{References}

Abdul-Razzak, H. and Ghan, S. J.: Parameterization of the influence of organic surfactants on aerosol activation, J. Geophys. Res. Atmos., 109(D3), D03205, doi:10.1029/2003JD004043, 2004.

Alfarra, M. R., Paulsen, D., Gysel, M., Garforth, A. A., Dommen, J., Prévôt, A. S. H., Worsnop, D. R., Baltensperger, U., and Coe, H.: A mass spectrometric study of secondary organic aerosols formed from the photoxidation of anthropogenic and biogenic precursors in a reaction chamber, Atmos. Chem. Phys., 6, 52795293, 2006,

http://www.atmos-chem-phys.net/6/5279/2006/.

Amundson, N. R., Caboussat, A., He, J. W., Martynenko, A. V., Savarin, V. B., Seinfeld, J. H., and Yoo, K. Y.: A new inorganic atmospheric aerosol phase equilibrium model (UHAERO), Atmos. Chem. Phys., 6, 975-992, 2006,

http://www.atmos-chem-phys.net/6/975/2006/.

Brooks, S. D., DeMott, P. J., and Kreidenweis, S. M.: Water uptake by particles containing humic materials and mixtures of humic materials with ammonium sulfate, Atmos. Environ., 38(13), 1859-1868, 2004

Chan, M. N., Choi, M. Y., Ng, N. L., and Chan, C. K.: Hygroscopicity of water-soluble organic compounds in atmospheric biomass burning derived organic species, Environ. Sci. Technol., 39, 1555-1562, 2005.

ChemSketch: Version 5.0. Advanced Chemistry Development, Inc., Toronto ON, Canada, www.acdlabs.com, 2003.

Clegg, S. L. and Pitzer, K. S.: Thermodynamics of Multicomponent, Miscible, Ionic-Solutions - Generalized Equations for
Symmetrical Electrolytes, J. Phys. Chem., 96(8), 3513-3520, 1992.

Clegg, S. L. and Seinfeld, J. H.: Thermodynamic models of aqueous solutions containing inorganic electrolytes and dicarboxylic acids at $298.15 \mathrm{~K}$. I. The acids as non-dissociating compounds, J. Phys. Chem. A, 110, 5692-5717, 2006.

Clegg, S. L., Seinfeld, J. H., and Brimblecombe, P.: Thermodynamic modelling of aqueous aerosols containing electrolytes and dissolved organic compounds, J. Aero. Sci., 32(6), 713-738, 2001.

Escobedo, J. and Mansoori, G. A.: Surface tension prediction for pure fluids, AIChE J., 42(5), 1425-1433, 1996.

Facchini, M. C., Decesari, S., Mircea, M., Fuzzi, S., and Loglio, G.: Surface tension of atmospheric wet aerosol and cloud/fog droplets in relation to their organic carbon content and chemical composition, Atmos. Environ., 34(28), 4853-4857, 2000.

Fainerman, V. B., Miller, R., and Aksenenko, E. V.: Simple model for prediction of surface tension of mixed surfactant solutions, Adv. Col. Int. Sci., 96(1-3), 339-359, 2002.

Fainerman, V. B. and Miller, R.: Simple method to Estimate Surface tension of Mixed Surfactant Solutions, J. Phys. Chem. B, 105, 11 432-11 438, 2001.

Fainerman, V. B., Wustneck, R., and Miller, R.: Surface tension of mixed surfactant solutions, Tenside Surfact Det., 38(4), 224-229, 2001.

Feingold, G.: Modeling of the first indirect effect: Analysis of measurement requirements, Geophys. Res. Lett., 30(19), ASC 7-1, 2003.

Fredenslund, A., Jones, R. L., and Prausnitz, J. M.: GroupContribution Estimation of Activity-Coefficients in Nonideal Liquid-Mixtures, AIChE J., 21(6), 1086-1099, 1975.

Fuzzi, S., Decesari, S., Facchini, M. C., Matta, E., Mircea, M., and Tagliavini, E.: A simplified model of the water soluble organic component of atmospheric aerosols, Geophys. Res. Lett., 28(21), 4079-4082, 2001.

Gaman, A. I., Kulmala, M., Vehkamaki, H., Napari, I., Mircea, M., Facchini, M. C., and Laaksonen, A.: Binary homogeneous nucleation in water-succinic acid and water-glutaric acid systems, J. Chem. Phys., 120(1), 282-291, 2004.

Goldsack, D. E. and White, B. R.: An Iterative Technique for Calculating Surface Tensions of Non Electrolyte Solutions, Canadian Journal of Chemistry-Revue Canadienne De Chimie, 61(8), 1725-1729, 1983.

Hansen, H. K., Rasmussen, P., Fredenslund, A., Schiller, M., and Gmehling, J.: Vapor-Liquid-Equilibria by Unifac Group Contribution, 5. Revision and Extension, Ind. Eng. Chem. Res., 30(10), 2352-2355, 1991.

Henning, S., Rosenorn, T., D’ Anna, B., Gola, A. A., Svenningsson, B., and Bilde, M.: Cloud droplet activation and surface tension of mixtures of slightly soluble organics and inorganic salt, Atmos. Chem. Phys., 5, 575-582, 2005, http://www.atmos-chem-phys.net/5/575/2005/.

Hori, M., Ohta, S., Murao, N., and Yamagata, S.: Activation capability of water solubile organic substances as CCN, Aerosol Sci., 34, 419-448, 2003.

Hu, Y. F. and Lee, H.: Prediction of the surface tension of mixed electrolyte solutions based on the equation of Patwardhan and Kumar and the fundamental Butler equations, J. Col. Int. Sci., 269(2), 442-448, 2004. 
Jasper, J. J.: The Surface Tension of Pure Liquid Compounds, J. Phys. Chem. Ref. Data, 1, 841-1010, 1972.

Kiss, G., Tombacz, E., and Hansson, H. C.: Surface tension effects of humic-like substances in the aqueous extract of tropospheric fine aerosol, J. Atmos. Chem., 50(3), 279-294, 2005.

Kokkola, H., Sorjamaa, R., Peräniemi, A., Raatikainen, T., and Laaksonen, A.: Cloud formation of particles containing humic-like substances, Geophys. Res. Lett., 33, L10816, doi:10.1029/2006GL026107, 2006.

Li, Z., Williams, A. L., and Rood, M. J.: Influence of soluble surfactant properties on the activation of aerosol particles containing inorganic solute, J. Atmos. Sci., 55, 1859-1866, 1998.

Li, Z. B. and Lu, B. C. Y.: Surface tension of aqueous electrolyte solutions at high concentrations - representation and prediction, Chem. Eng. Sci., 56(8), 2879-2888, 2001.

Loglio, G., Pandolfini, P., Miller, R., Makievski, A. V., Ravera, F., Ferrari, M., and Liggieri, L.: Drop and Bubble Shape Analysis as a Tool for Dilational Rheology Studies of Interfacial Layers, in: Novel Methods to Study Interfacial Layers, Studies in Interface Science, edited by: Miller, D. M. a. R., Elsevier, Amsterdam, pp. 439-485, 2001.

Marcolli, C., Luo, B. P., and Peter, T.: Mixing of the organic aerosol fractions: Liquids as the thermodynamically stable phases, J. Phys. Chem. A, 108(12), 2216-2224, 2004.

Marrero, J. and Gani, R.: Group-contribution based estimation of pure component properties, Fluid Phase Equilib., 183, 183-208, 2001.

McFiggans, G., Artaxo, P., Baltensperger, U., Coe, H., Facchini, M. C., Feingold, G., Fuzzi, S., Gysel, M., Laaksonen, A., Lohmann, U., Mentel, M., Murphy, D. M., O’Dowd, C. D., Snider, J. R., and Weingartner, E.: The effect of physical and chemical aerosol properties on warm cloud droplet activation, Atmos. Chem. Phys., 6, 2593-2649, 2006,

http://www.atmos-chem-phys.net/6/2593/2006/.

Metzger, S., Dentener, F., Pandis, S., and Lelieveld, J.: Gas/aerosol partitioning: 1. A computationally efficient model, J. Geophys. Res. Atmos., 107(D16), 4312, doi:10.1029/2001JD001102, 2002.

Ming, Y. and Russell, L. M.: Thermodynamic equilibrium of organic-electrolyte mixtures in aerosol particles, AICHe J., 48(6), 1331-1348, 2002.

Needham, D.E., Wei, I.C. and Seybold, P.G,. Molecular Modeling of the Physical-Properties of the Alkanes. J Am Chem Soc, 110(13): 4186-4194. 1988

Peng, C., Chan, M. N., and Chan, C. K. The hygroscopic properties of dicarboxylic and multifunctional acids: Measurements and UNIFAC predictions, Environ. Sci. Technol., 35(22), 44954501, 2001.

Perry, R. H. and Green, D. W.: Perry's Chemical Engineers Handbook (7th edition), McGraw-Hill, 1997.

Poling, B. E., Prausnitz, J. M., and O'Connell, J. P.: The Properties of Gases and Liquids, McGraw-Hill Professional, 2000.

Quayle, O. R.: The parachors of organic compounds, Chem. Rev., 53, 439-591, 1953.

Reinhard, M. and Drefahl, A.: Handbook for Estimating Physicochemical Properties of Organic Compounds, John Wiley \& Sons, 1999.

Riddick, J. A., Bunger, W. B., and Sakano, T. K.: Techniques of Chemistry, Organic Solvents, J. Wiley and Sons, New York, pp. 553, 1986.

Rissman, T. A., Nenes, A., and Seinfeld, J. H.: Chemical amplification (or dampening) of the Twomey effect: Conditions derived from droplet activation theory, J. Atmos. Sci., 61(8), 919-930, 2004.

Rowlinson, J. S., Widom, B., Abraham, D. B., Evans, R., Sullivan, D. E.,,, Schofield, P., Henderson, J. R., Percus, J. K., Sluckin, T. J., Richmond, P., Findenegg, G. H., Rowlinson, J. S., Schofield, P., Pethica, B. A., Mclure, I. A., Rice, S. A., Smith, E. B., Mazo, R. M., Vassilieff, C. S., Whittington, S. G., Sluckin, T. J., Rasaiah, J. C., Develyn, M. P., Stepto, R. F. T., Croxton, C. A., Lal, M., Oxtoby, D. W., Vincent, B., Everett, D. H., Parsons, R., Parsonage, N. G., Blum, L., Nicholson, D., Torrie, G., Outhwaite, C. W., Levine, S., Bushnellwye, G., and Toxvaerd, S.: Structure of the Interfacial Region - General Discussion. Faraday Symp. Chem. S, 16, 205-256, 1981.

Seidl, W.: Model for a surface film of fatty acids on rain water and aerosol particles, Atmos. Environ., 34(28), 4917-4932, 2000.

Seinfeld, J. H. and Pandis, S. N.: Atmospheric Chemistry and Physics, Wiley-Interscience Publication, 1998.

Shulman, M. L., Jacobson, M. C., Charlson, R. J., Synovec, R. E., and Young, T. E.: Dissolution behavior and surface tension effects of organic compounds in nucleating cloud droplets (Vol. 23, p. 277, 1996), Geophys. Res. Lett., 23(5), 603-603, 1996.

Sorjamaa, R., Svenningsson, B., Raatikainen, T., Henning, S., Bilde, M., and Laaksonen, A.: The role of surfactants in Kohler theory reconsidered, Atmos. Chem. Phys., 4, 2107-2117, 2004, http://www.atmos-chem-phys.net/4/2107/2004/.

Sorjamaa, R. and Laaksonen, A.: The influence of surfactant properties on critical supersaturations of cloud condensation nuclei, J. Aerosol Sci., 37, 12, 1730-1736, 2006.

Sprow, F. B. and Prausnitz, J. M.: Surface Tensions of Simple Liquid Mixtures, Transactions of the Faraday Society, 62(521P), 1105-1111, 1966a.

Sprow, F. B. and Prausnitz, J. M.: Surface Tensions of Simple Liquids. Transactions of the Faraday Society, 62(521P), 1097-1104, 1966b.

Stanton, D. T. and Jurs, P. C.: Development and Use of Charged Partial Surface-Area Structural Descriptors in Computer-Assisted Quantitative Structure Property Relationship Studies, Anal. Chem., 62(21), 2323-2329, 1990.

Suarez, J. T., Torres-Marchal, C., and Rasmussen, P: Prediction of surface tension of nonelectrolyte solutions, Chem. Eng. Sci., 44(3), 782-786, 1989.

Svenningsson, B., Rissler, J., Swietlicki, E., Mircea, M., Bilde, M., Facchini, M. C., Decesari, S., Fuzzi, S., Zhou, J., Monster, J., and Rosenorn, T.: Hygroscopic growth and critical supersaturations for mixed aerosol particles of inorganic and organic compounds of atmospheric relevance, Atmos. Chem. Phys., 6, 1937-1952, 2006 , http://www.atmos-chem-phys.net/6/1937/2006/.

Tamura, M., Kurata, M., and Odani, H.: Practical Method for Estimating Surface Tensions of Solutions, Bulletin of the Chemical Society of Japan, 28(1), 83-88, 1955.

Topping, D. O., McFiggans, G. B., and Coe, H.: A curved multicomponent aerosol hygroscopicity model framework: Part 1 - Inorganic compounds, Atmos. Chem. Phys., 5, 1205-1222, $2005 a$. 
Topping, D. O., McFiggans, G. B., and Coe, H.: A curved multicomponent aerosol hygroscopicity model framework: Part $2-$ Including organic compounds, Atmos. Chem. Phys., 5, 1223$1242,2005 b$.
Tuckermann, R. and Cammenga, H. K.: The surface tension of aqueous solutions of some atmospheric water-soluble organic compounds, Atmos. Environ., 38(36), 6135-6138, 2004. 